\title{
La dimensión sectorial del cooperativismo colombiano a través de un análisis de redes
}

\author{
Miguel Ángel Alarcón Conde \\ Juan Fernando Álvarez
}

RESUMEN: Entre 2003 y 2010 se produce en Colombia un incremento sin precedentes en la constitución y actividad productiva de cooperativas, alcanzando un peso muy notable en la economía. Posteriormente, la regulación de las Cooperativas de Trabajo Asociado supone la desaparición de un número muy importante de ellas, aspecto que explica la acotación temporal del trabajo. Esa acotación también se explica por las limitaciones en la disponibilidad de información, agravadas por la desaparición del Centro de Investigación del Cooperativismo de Colombia y la consecuente desaceleración en las investigaciones sobre el tema, una vez cancelado su papel como principal usuario y poseedor de datos desagregados del cooperativismo.

Con todo, los efectos de arrastre del cooperativismo colombiano, en términos intersectoriales y de empleo, tienden a ser menores y generan más empleo indirecto relativamente a los de la economía nacional ante incrementos de la demanda, suponiendo que el cooperativismo tiene una desventaja competitiva respecto a la estructura productiva nacional, más especializada en sectores de demanda débil-media. Además, a través de un Análisis de Redes Sociales, las cooperativas se adaptan al periodo de expansión económica aumentando la cohesión de sus redes intersectoriales, pero se tienen efectos contrarios en fases recesivas.

En consecuencia, el sector cooperativo colombiano ha contribuido al desarrollo económico y social, y actualmente busca optimizar sus funciones como organizador del territorio y mediador de la convivencia pacífica. Así, los estudios sobre impacto sectorial y sobre la evaluación de impacto de Políticas Públicas son un elemento indispensable para garantizar la construcción compartida de políticas públicas entre los emprendimientos cooperativos, y solidarios, la Academia y el Gobierno.

PALABRAS CLAVE: Análisis de Redes Sociales, Sistemas de Cuentas Nacionales, Cooperativismo, Evaluación del Impacto, Análisis Sectorial, Políticas Públicas, Colombia.

CLAVES ECONLIT: C65, C67, E24, J54, P13.

Cómo citar este artículo / How to cite this article: ALARCÓN, M.A. \& ÁLVAREZ, J.F. (2018): "La dimensión sectorial del cooperativismo colombiano a través de un análisis de redes", CIRIEC-España, Revista de Economía Pública, Social y Cooperativa, 92, 121-154.

Correspondencia: Miguel Ángel Alarcón Conde, Doctor en Economía, Universidad de Castilla-La Mancha, email: miguelangel.alarcon@uclm.es; Juan Fernando Álvarez, Doctor en Ciencias Sociales, Pontificia Universidad Javeriana, alvarez_juan@javeriana.edu.co. 


\section{EXPANDED ABSTRACT}

\section{The sectoral dimension of Colombian cooperativism through a Social Network Analysis}

Context. An unprecedented increase in the constitution and productive activity of cooperatives took place in Colombia in 2003-2010 period, reaching a very significant weight in the economy. Subsequently, the regulation of the Associated Work Cooperatives supposed the disappearance of a very important number of them, that it explains the temporary delimitation of this paper. This period was also limited by the availability of information, which was aggravated by the disappearance of the Center for Research on Cooperativism of Colombia and the consequent deceleration in the investigations on the issue because of it was the main user and possessor of disaggregated data of cooperativism.

However, according to the comparative information that has been consulted for the Colombian economy and its cooperative segment, the cooperatives were more than 8,500 , which paid compensations to 140,000 employees; they gave work to almost 540,000 people if the workers of Associated Work Cooperatives were included, and they were generating of just over Colombian Pesos 8.5 trillion GDP - 12 digits-. This assumed 0.4 percent of the active entities in Colombia, according to the data from CONFECOOP and the DANE's Annual Survey of Manufacturing, which represented almost 1.5 percent of employees in Colombia, proportion that rose up 3 percent if the employees in Associated Work Cooperatives were considered. At the same time, cooperativism GDP reached almost 1.7 percent of the national GDP, starting in 2003 with the half of that participation.

Thus, it is showed the remarkable importance of the cooperative sector in the Colombian economy, which achieved very similar sectoral proportions of the national employment and entities as the case of Mining branches, that weighted half of the GVA generated by Energy or Financial Intermediation sectors in the Colombian economy -3.5 and 5.1 percent of the Colombian GVA, respectively- and they assumed 1.2 percent of total entities and 1.1 percent of total employment. In parallel, cooperativism employees of the Construction branches were 5 percent of the Colombian employees. These aspects were technical consequences of the productive specialization of the Colombian cooperativism services sectors in production, entities and employment, such as Financial Intermediation, Education, Health and Social Services, which also increased their specialization index. 


\section{LA DIMENSIÓN SECTORIAL DEL COOPERATIVISMO COLOMBIANO A TRAVÉS DE UN ANÁLISIS DE REDES}

Methods. It is possible to study what extent it is possible to create an intersectoral relations network as a centralized structure through the Social Networks Analysis. On this issue, three indicators bring us the required knowledge: density, the percentage of the links over the possible -total- relationships; cohesion, defined in theory of networks as the effort to relate between nodes, what determines the number of relationships on the shortest path between two nodes; and the degree of centralization -Network Centralization Index, $\mathrm{NCl}$ - which is the variability in degrees as a percentage of the maximum centralization; that is to say, in relation to the star network structure, that shows the maximum power of a single productive sector.

Graph Theory principles allow us to observe the differences between the national and cooperativism networks (inter-sectoral relations) in order to determine the power of certain productive sectors or the meaning of the centrality and centralization indicators in them. Always under a different approach, which could enrich the conventional interpretation with the (Social Networks) Graphs and their indicators.

In addition, the intermediate position of a productive sector between other sectors means their power over the transactions between non-adjacent branches; that is to say, counting relations between two sectors not directly related. Thus, the indicator reflects the power of the mostly intermediary productive branch, either to spread their beneficial or damaging effects. The Financial Intermediation is a good example of a sector with intermediary power, which would reach alternative consequences according to growth or recessive dynamics.

Results and future research. On a sector basis, the Colombian cooperativism was concentrated in Education, Health and Other Personal and Community Services, Financial Intermediation and Trade and Hospitality: they obtained almost 75 percent of GVA and employment, while the same national sectors contributed to those indicators between 40 and 50 percent. Moreover, the cooperative concentration grew in Manufacturing, Financial Intermediation and Education, Health and Other Personal and Community Services.

Furthermore, if it is compared the structures of intersectoral relations between total economy and cooperativism in the period we find some differences although the motor branches are the same: Manufacturing, Construction, Trade and Hospitality and Transport and Communications. However, the cooperative sectors were adapted to business cycle and increased their participations, especially in Trade and Hospitality branches.

In parallel, the cooperatives of the Financial Intermediation sector had -and they have- a considerable weight, both in the cooperativism and in the same national sector. Moreover, they increased their participations, both in the cooperativism and in the national sectors, and they maintained their backward and forward effects below the average of the Cooperative sector, as the case of the national Financial Intermediation sector. 
The effects of the cooperativism in that period, either on employment or on inter-sectoral terms, were lower than the same effects on the national economy, and they were generating more indirect employment than the same national sectors, in response to the increases in demand. That might imply that cooperativism it had a competitive disadvantage with respect to the national productive structure, which was more specialized in weak-medium demand sectors. In addition, under the Social Networks Analysis, cooperatives were adapted to the economic expansion by increasing the cohesion of their inter-sectoral graphs, so they will have opposite effects in recessive phases, with the exception of Electricity, Gas and Water Supplies, Construction, Trade and Hospitality and Financial Intermediation, the branches which cooperativism achieved the greater proportion of the direct employment.

The graphs of the cooperativism -their inter-sectoral relations- were more centralized structures than the national economy ones because of Manufacturing, Financial Intermediation and the Agricultural sector. Whereas Manufacturing, Business Services, Agricultural sector and Transport and Communications -a key sector in the dynamics of globalization- centralized the network of the national economy, which did not and still does not have the same role in the cooperative graphs. Consequently, the graphs that represented the networks of the Colombian national economy were more dense and cohesive than cooperative ones, while they were evolving, which meant a certain convergence between them.

In short, the adaptation of the Colombian cooperativism to the economic and social development stage, between 2003 and 2010, supposed cohesion of its networks, although some differences is maintained in the productive core of the national economy based on the quantity responses to the business cycle.

All the foregoing figures redounds in a good help to support the initial studies of the impact of cooperativism on the national economy, particularly to promote the strengthening of peaceful coexistence in Colombia. Consequently, the Colombian cooperatives are seeking to optimize their possible functions as organizers of the territory and mediators of peaceful coexistence. Thus, the studies on sectoral impact and on the impact evaluation of public policies will need to know many more social and economic indicators as essential elements to ensure a construction of public policies bringing together Cooperatives, Solidarity Organizations, Academy and Government.

KEYWORDS: Social Networks Analysis, System National Accounts, Cooperativism, Impact Evaluation, Sectoral Analysis, Public Policies, Colombia. 


\section{LA DIMENSIÓN SECTORIAL DEL COOPERATIVISMO COLOMBIANO A TRAVÉS DE UN ANÁLISIS DE REDES}

\section{Introducción}

Tras el desarrollo del proyecto de investigación (Proyecto-2013, en adelante) denominado Impacto Social y Económico del Cooperativismo en Colombia, auspiciado por el Centro de Investigación del Cooperativismo de Colombia (CENICOOP, en adelante), que anunció sus documentos de trabajo fundamentales en la segunda mitad de 2013, y la posterior presentación, en 2016, de algunos de los resultados del Informe CENICOOP-2013 (Alarcón, 2013), auspiciado por CIRIEC-Colombia ${ }^{1}$, ante la Unidad Administrativa Especial de Organizaciones Solidarias (UAEOS) en presencia de su Dirección Nacional2, los actores coincidieron en la importancia de contar con referentes metodológicos para el abordaje de un asunto de vital interés para el sector como su consistencia y vertebración.

No obstante, el rigor de distintas coyunturas bien sea la desaparición del CENICOOP como órgano de investigación, la ausencia de una línea de indagación asentada en las distintas Universidades que hacen parte del conglomerado de instituciones que estudian el tema en Colombia, la focalización de los entes gremiales y de promoción gubernamental en el papel del cooperativismo en la nueva coyuntura de paz y los vaivenes tributarios, han dejado al tema como un referente de lo posible pero con pocos avances fácticos.

Ante la necesidad de generar acciones de convivencia pacífica en los territorios, de cuantificar el aporte de las cooperativas y de proyectar nuevas estrategias de fortalecimiento sectorial en un marco de creciente participación del sector cooperativo en la co-construcción de políticas públicas, el tema de la evaluación del impacto de las políticas públicas sobre el cooperativismo vuelve a tener una relevancia sustantiva.

Así, entre otros objetivos, este trabajo intenta dar a conocer el estudio de economía aplicada mencionado al inicio de esta Introducción y extenderlo para analizar ciertos indicadores económicos del cooperativismo resultados del empirismo, con el fin de poner a discusión su utilidad como línea base orientada a fortalecer los estudios de impacto, sus potencialidades y restricciones como mecanismos de depuración y cuyo resultado final se enmarca en su potencial refutación, idea principal que se quiere destacar.

El trabajo se desarrollará como sigue. Se inicia con un epígrafe metodológico para justificar el periodo a tener en cuenta en el trabajo, es decir, 2003-2010, así como otros aspectos relacionados con magnitudes, sectores y el contexto nacional obtenidos de la cuantificación del circuito económico coopera-

1.- Centro Internacional de Investigación e Información sobre la Economía Pública, Social y Cooperativa.

2.- En un encuentro el $27 / 07 / 2016$ en el que se discute la posibilidad de un Convenio de colaboración para un Observatorio sobre Economía Social y Solidaria para Colombia en la sede de la UAEOS con la Universidad Católica de Colombia y miembros del CIRIEC-Colombia. 
tivo colombiano en el contexto del proyecto Impacto Social y Económico del Cooperativismo en Colombia, entendido a través de la sucesión de las Cuentas Satélite, en perspectiva comparada con el circuito económico nacional. Seguidamente, se exponen unas referencias a la concentración cooperativa de Colombia por sectores, número de entidades, y empleo. A continuación, se presenta un ejercicio de economía aplicada en el que se ofrecen resultados preliminares de un análisis de las redes de relaciones intersectoriales del Cooperativismo en perspectiva comparada con las propias redes intersectoriales de la economía de Colombia con el objetivo de observar la dinámica de su cohesión, densidad y centralidad, de las que se destacarán sus diferencias estructurales. El ejercicio tiene como base las Tablas Input Output (TIO, en lo sucesivo) del cooperativismo colombiano obtenidas de Alarcón (2013). Finalmente, se analiza la importancia de una línea base para el desarrollo de estudios de impacto en la actual coyuntura colombiana tanto como eje de orientación de estrategias de desarrollo como para elemento de co-construcción de políticas públicas. Unas conclusiones cierran el trabajo.

\section{Métodos y fuentes. Perímetro estimativo del cooperativismo de Colombia en términos cuantitativos}

En el año 2013 el CENICOOP desarrolló la Metodología CENICOOP-2013 (Alarcón, Castro y Rodríguez, 2013). La metodología fue un referente en la construcción de las Cuentas Satélite del cooperativismo colombiano para elaborar el Informe CENICOOP-2013 (Alarcón, 2013). La presentación de la serie temporal de Cuentas Agregadas del segmento cooperativo colombiano es una de las más largas del mundo con respecto a variables y datos (a fecha de aceptación de este trabajo) y el análisis de economía aplicada sobre la depuración de información del cooperativismo colombiano más abarcador que se haya realizado.

Posteriormente, Rodríguez, Sánchez, Álvarez y Fajardo (2016) presentan un aporte hacia un reporte de variables sociales, de tipo mesoeconómico, al que se le anexan ciertas cifras agregadas, al modo del Proyecto 2013, pero el ejercicio queda pendiente elevar los datos a las cifras poblacionales, en tanto que el Sistema Sigcoop y los datos potenciales obtenidos de CONFECOOP lo permitía (en tanto era posible a través de CENICOOP). También integra indicadores cuantitativosmonetarios, asumidos por la ortodoxia, con los de tipo cualitativo, de corte más heterodoxo, que pueden dar más información sobre los resultados sociales, también de gestión social (que, en sentido amplio, incluiría las tendencias de estudio sobre Memorias de Sostenibilidad, la Economía Circular 0 la Economía del Bien Común) y la propia elaboración de Balances Sociales por parte de las cooperativas, pero no alcanza a la estimación del perímetro en el que situar grupos, sectores o clusters de las organizaciones implicadas.

La obra de Rodríguez, et al. (2016), por tanto, es una incipiente propuesta de cálculo de impacto socioeconómico, que sólo tendría resultados definitivos siempre que haya acuerdo entre el conjunto 


\section{LA DIMENSIÓN SECTORIAL DEL COOPERATIVISMO COLOMBIANO A TRAVÉS DE UN ANÁLISIS DE REDES}

de los gremios cooperativos para realizar Balances Sociales, decidir estándares claros (conscientes de la diversidad de criterios válidos) y brindar información a la Sociedad Civil, a la Academia y a la Administración: cuestiones costosas en tiempo y dinero, a la par que en recelo de las entidades y de las Administraciones Públicas por su tráfico. Se trata de una paradoja, cuando lo que se supone es que las cooperativas deberían dar a conocer sus actividades e impacto social y, al tiempo, que esas entidades se situaran en el marco de unas grandes cifras económicas, a nivel nacional, de empleo, 0 de transferencias sociales, entre otras.

Por tanto, esa propuesta de indicadores socioeconómicos no termina de plasmarse en indicadores sintéticos, ni la mayor parte de los indicadores propuestos son posibles de obtener debido a que no hay suficiente información recopilada para la, también, propuesta necesaria de sondeos que debe incluir. Quizás daría una mayor robustez a la propuesta la incorporación de indicadores sintéticos, corregidos por la elevación a la población, o la incorporación de referencias, sean indicadores medianos, sean de densidad de las actividades de promoción social o también correcciones sobre errores de muestreo, siempre en estudios posteriores para un productivo feedback. No obstante, se insiste, que la aplicación preliminar que lleva inmersa esa obra es el intento más reciente de cuantificación económica y social del cooperativismo en Colombia.

En definitiva, las propuestas de medición del impacto social y económico del cooperativismo en Colombia, como la obra de Rodríguez, et al. (2016), se ven limitadas por el acceso a la información y en ello buscan insistir en que las entidades estatales den cumplimiento a su obligación legal de ofrecer estadística a la Sociedad Civil, Administración y estudiosos, para permitir conocer la realidad de las cooperativas y su entorno.

\subsection{Limitaciones de la información}

En el trasfondo de la información cooperativa en Colombia subyacen disonancias cognitivas frente a las diversas concepciones de las instituciones que recolectan información, así como en la ausencia de mecanismos estandarizados de solicitar, de la sistematización de la información y del lapso entre el periodo de recolección y de publicación. Además, el registro de los diferentes tipos de cooperativas está dispersa en varias instituciones públicas y privadas, las cuales registran la información según sus necesidades e intereses creando asimetrías de información.

En lo que se refiere a cuentas nacionales, la entidad encargada de recoger la información estadística es el Departamento Administrativo Nacional de Estadística (DANE), y no tiene un mecanismo claro de tratamiento estadístico para las cooperativas. En algunos de sus estudios, introducen la noción de instituciones sin fines de lucro (ISFL), las cuales se considera pueden ser de mercado y de no mercado, y se clasifican en instituciones sin fines de lucro al servicio de los hogares (ISFLSH), instituciones sin fines de lucro financiadas por el gobierno e instituciones sin fines de lucro que sirven a las empresas (ISFLSE) (DANE, 2005), propios del sistema de cuentas nacionales de Naciones Unidas. 
Sin embargo, el tema fue planteado en el año 2005 y no ha sido desarrollado, sino que se ha quedado en materia de discusión y estudios (DANE, 2008). Todo ello en el entorno de las Cuentas Nacionales, planteadas por el propio organismo desde 1986 (DANE,1986). Por otra parte, la Dirección de Impuestos y Aduanas Nacionales, DIAN, captura información de contribuyentes especiales, entre los que figuran las cooperativas. Las variables de reporte son fundamentalmente contables y financieras, bajo las cuales examina liquidaciones y presunción de rentas, patrimonio e impuestos liquidados y por liquidar. Esta información no es de carácter público y su acceso requiere el paso por múltiples filtros burocráticos.

A lo anterior se añade la Superintendencia de Economía Solidaria ${ }^{3}$, que captura información de aquellas cooperativas que son vigiladas por esta entidad y pone el énfasis en los instrumentos financieros que en la actualidad son utilizados para garantizar el cumplimiento de su normatividad. Tanto la Superintendencia de la Economía Solidaria como la Dirección de Impuestos y Aduanas Nacionales hacen parte del Ministerio de Hacienda y Crédito Público. De igual forma, hay información de otras Superintendencias que también vigilan a cooperativas específicas, como es el caso de la Superintendencia de Transporte y Superintendencia de Servicios Públicos adscrita al Departamento Nacional de Planeación; la Superintendencia Nacional de Salud, adscrita al Ministerio de Salud; la Superintendencia de Puertos y Transportes adscrita al Ministerio de Transporte y la Superintendencia de Vigilancia y Seguridad Privada adscrita al Ministerio de Defensa. Como cada organización tiene criterios y períodos distintos para registrar las cifras de las cooperativas, hay problemas para agregar la información.

Así, cuando de información de cooperativas existentes en un año determinado se trata, los diferentes registros disponibles tienen un error que termina sesgando la información: los registros inician con la creación de las cooperativas y suponen la vida asociativa a partir de la actualización de datos financieros. En adición, la desagregación de información por tipología organizacional presenta problemas de agregación. Por ejemplo, la información estadística de las cooperativas de consumo está diseminada, según el año en que se revise la información, en una categoría denominada consumo, distribución y consumo o dentro de las estadísticas agregadas de otros subsectores.

De manera que si, en un período determinado, una cooperativa no reporta estados financieros, la información de su permanencia suele tomarse con base en los registros anteriores y sólo ante la persistencia de la desinformación por más de cinco años termina siendo retirada de los registros. De manera que los registros de información reportan la presunción de vida cooperativa, pero no registran la salida por factores tangibles como la liquidación o inactividad de una empresa.

En cuanto a información sobre los asociados, no hay mecanismos que permitan verificar la vida asociativa del asociado en cuestión, ni tampoco su posible adhesión a otras cooperativas. En muchos

3.- Organismo colombiano de control y supervisión del sector de la economía solidaria, el cual incluye cooperativas, fondos de empleados, asociaciones mutuales y unas pocas organizaciones asociativas de naturaleza autogestionaria. 


\section{LA DIMENSIÓN SECTORIAL DEL COOPERATIVISMO COLOMBIANO A TRAVÉS DE UN ANÁLISIS DE REDES}

casos, las cooperativas cuentan con un número de asociados inhábiles o que simplemente rezagan sus actuaciones con la cooperativa, hasta hacerlas igual a cero. Estos asociados no suelen participar de la gestión de la organización, y se carece de compromiso con una entidad de naturaleza autogestionaria. Otro caso similar es el de asociados que disfrutan los beneficios de la cooperación, pero no están dispuestos a pagar por ellos. Sea cual sea el caso, no existen mecanismos para verificarlos estadísticamente y se genera un número de asociados posiblemente mayor a los que tienen vida asociativa. Cuando de empleos se trata, se han registrado casos donde los asociados de cooperativas de trabajo asociado reportan sus membresías, a la vez que su condición de trabajadores. Puede esto generar duplicidades que afectan el agregado de la información.

Además, en el ámbito de la agregación estadística existen tres fuentes distintas, que presentan información desarticulada entre sí en cuanto a períodos, organizaciones y desagregaciones distintas. La primera fuente de información proviene del registro de las entidades sin ánimo de lucro 4 en el registro único de empresas sociales de las Cámaras de Comercio. En esta fuente se registra regularmente el nacimiento, y eventualmente la actualización de información, pero no hay mecanismos para verificar su vida operativa.

Otra fuente de información proviene del registro de cooperativas en una base de datos diseñada desde la Confederación de Cooperativas de Colombia, pero que por sustracción de contenidos no logró el reporte periódico de las organizaciones y se ha transferido a los entes estatales de supervisión. La tercera fuente presenta la información de las entidades que le corresponde supervisar y controlar a la Superintendencia de Economía Solidaria, la cual registra un universo parcial.

Ello comporta, desde el punto de vista metodológico, varios problemas, y hace que la investigación sea costosa generando que la agregación de conocimiento se dificulte. Sin embargo, la profundización desde varias ópticas demuestra que los problemas transversales siguen siendo los mismos y permite identificar vacíos conceptuales que adolecen de propuestas. Algunas propuestas han sido expuestas por autores como Andrea y Vincenti (2015), quienes plantean que la evaluación del valor añadido social que producen las cooperativas genera cambios en las dimensiones de vida social a nivel micro, meso y macro, identificando para las dos últimas los resultados en términos de bienes relacionales y capital social. Partiendo de una clasificación similar Álvarez en Rodríguez et al. (2015) plantea que la dimensión micro de las cooperativas puede verificarse en términos de beneficios transferidos a los asociados, mientras que a nivel meso apela al balance social para su medición y a nivel macro de las cuentas nacionales. Sea cual sea la propuesta es menester contar con fuentes de información confiables que permitan avanzar en la generación de líneas de base que permitan ejercicios comparativos y de determinación de variables de mayor peso en el impacto cooperativo.

4.- Que involucra, además de las empresas solidarias como cooperativas, fondos de empleados y asociaciones mutuales, a un universo de organizaciones mucho mayor, como fundaciones, corporaciones, voluntariados y otro tipo de asociaciones civiles, comunitarias, políticas y religiosas, entre otras. 


\subsection{Antecedentes de medición de las cifras del cooperativismo en Colombia}

Aunque se han realizado propuestas sobre construcción de un circuito económico agregado del cooperativismo en Colombia, como las presentadas por Rodríguez (1993) y por Bernal (2008), hasta el momento no se ha hecho una cuantificación real del aporte del sector solidario a la economía 5 , entendido como el que añade las ISFL y otras no certificadas de impacto social y sostenibles ambientalmente, entre otras, al segmento cooperativo. A pesar de ello, se plantea en el Proyecto-2013 la iniciativa de aplicar del Sistema de Cuentas Nacionales (SCN, en lo sucesivo) para las cooperativas con el fin de conocer aspectos como su producción, valor agregado, remuneración a los asalariados, excedente bruto de explotación, consumo de capital fijo, ingreso disponible, formación bruta de capital fijo y la necesidad o capacidad de financiación del sector; con la finalidad de estudiar tanto su evolución en el tiempo (dinamismo) como su representatividad en la economía nacional. Por tanto, teniendo en cuenta que las cooperativas participan en diferentes actividades económicas, también se consideró importante construir el SCN por sector de actividad, reflejando así los más importantes para el sector cooperativo y donde aportan más al total de la economía nacional. No obstante, no era materialmente posible llevarlo al sector de la Economía Social y Solidaria en sentido amplio.

La Metodología- $2013^{6}$ se diseñó siguiendo el estándar particularizado del DANE para construir las Cuentas de los Sectores Institucionales en Colombia, la cual consiste en la homologación de los estados financieros a las cuentas que conforman el SCN. También se tuvieron en cuenta, especialmente, el Manual para la Elaboración de las Cuentas Satélite de las Empresas de la Economía Social: Cooperativas y Mutuas, que elaboró el Centro Internacional de Investigación e Información sobre la Economía Pública, Social y Cooperativa (Barea y Monzón, 2006); la obra pionera de Naciones Unidas Handbook on Non-Profit Institutions in the System of National Accounts (United Nations, 2003), abriendo las posibilidades de una futura integración en las Cuentas Satélite de las ISFL en términos amplios; así como los trabajos de Rodríguez (1993) y Bernal (2008).

Con la información comparativa para la economía nacional y el segmento cooperativo tanto del DANE, como de CONFECOOP y CONFECÁMARAS, y las estimaciones resultado de la Metodología2013, se señala que las cooperativas de la economía colombiana en 2010 constituyen más de 8.500 entidades, que remuneraron a unos 140.000 asalariados y dieron trabajo a casi 540.000 empleos dependientes y no dependientes, si se contabilizan los trabajadores asociados a CTA, según los repor-

5.- Salvo, de manera limitada, la Metodología-2013 para el cooperativismo, al utilizar «los estados financieros anuales a 6 dígitos que reportan las entidades de economía solidaria a la Superintendencia de la Economía Solidaria y a la Superintendencia de Puertos y Transporte, a través de la Confederación de Cooperativas de Colombia (CONFECOOP), los cuales están disponibles para el período 2002-2011, a mediados de 2013. Debido a que existen entidades que no hacen un reporte continuo de sus estados financieros, se seleccionaron las entidades que sí reportan continuamente, bajo el criterio de que hubiesen reportado en solo un año o en dos o más períodos seguidos. Las entidades que tienen vacíos en sus reportes, no se tuvieron en cuenta. La clasificación por actividad económica se realizó utilizando la Clasificación Internacional Industrial Uniforme Revisión 3 (CIIU Rev. 3). Las entidades se clasificaron, en la medida de lo posible, teniendo en cuenta su mayor ingreso operacional» (Alarcón, Castro y Rodríguez, 2013).

6.- La Metodología-2013 se puede aplicar a cooperativas, fondos y mutuales en Colombia, pero sólo contempla los cálculos para las cooperativas y, en consecuencia, en este trabajo sólo será aplicado a este subsector de la economía solidaria. 


\section{LA DIMENSIÓN SECTORIAL DEL COOPERATIVISMO COLOMBIANO A TRAVÉS DE UN ANÁLISIS DE REDES}

tes remitidos a CENICOOP, y generan un valor añadido anual de poco más de 8,5 billones de pesos colombianos -millones de millones-. Esto viene a suponer, por un lado, el 0,4 por 100 de las entidades consideradas como activas en Colombia, a tenor de las cifras deducidas de las informaciones de CONFECOOP y la Encuesta Anual Manufacturera del DANE7 . Por otro lado, el sector cooperativo representa casi el 1,5 por 100 del empleo asalariado en Colombia, proporción que se eleva al 3 por 100 si se considera el trabajo aportado por los asociados en CTA. Paralelamente, el PIB cooperativo -VAB con el ajuste de los Impuestos netos a la producción- alcanza casi el 1,7 por 100 del nacional, partiendo en 2003 de la mitad de esa participación (Cuadro 1).

Como se desprende del cuadro 1, el período 2003-2010 fue para el cooperativismo colombiano un período de expansión, pero también para la economía nacional. Según datos del Banco de la República (2018), el PIB per cápita de la economía colombiana a precios nominales tiene un crecimiento acumulado entre el año 2003 y 2010 que hace duplicar su PIB, y casi (88 por 100) su PIB por habitante. Crecimientos, en buena parte, basados en la apertura de nuevos tratados de libre comercio impulsados por el Gobierno de Álvaro Uribe, la intensificación de las concesiones mineras y una balanza comercial positiva en un contexto de altos precios del petróleo y apreciación de la moneda. Sin embargo, pese a que el país creció, la tasa de desigualdad se mantuvo mayor al promedio de América Latina, bajando levemente de 0,58 en 2003 a 0,56 en 2010, el índice de ocupación permaneció casi constante, en torno al 55 por 100, mientras que el desempleo disminuyó notablemente en 5,6 puntos porcentuales de la población activa, del 16,7 por 100 al 11,1 por 100.

En este período hubo un importante crecimiento de las CTA, que terminaron desapareciendo en una gran medida. Hay evidencia que demuestra que el crecimiento vino aparejado con una política de instrumentalización estatal para disminuir costes laborales y reducir la nómina de organismos públicos y entidades privadas (Álvarez y García, 2013). En paralelo se actualizan las normas de supervisión y fiscalización de las cooperativas financieras (Ley 795 de 2003), haciéndolas más cónsonas con las estrategias de internacionalización que seguía el gobierno nacional. Por otra parte, se establecen normas aduaneras y fiscales en las que las cooperativas son asimiladas a empresas capitalistas del mismo ramo (Leyes 788 de 2002 y 863 de 2003), asimismo y se decretan normas de gestión del riesgo de liquidez en cooperativas de ahorro y crédito.

Para el año 2004, se impone una contribución impositiva del 20\% de los excedentes de las cooperativas dirigida a la financiación de la educación pública formal (Decreto 2880 de 2004). Posteriormente, hacia 2008, el fenómeno de instrumentalización de las CTA es advertida por la academia y los gremios sindicales y de cooperativismo, planteándose que la misma es una suerte de dum-

7.- No consta para Colombia un directorio o estadística de esas cifras se entiende directorio como registro numérico, no como registro del que obtener una serie de informaciones para su uso comercial, de negocios o relacional , lo cual provoca que el país o el estudioso no sepa exactamente cuántos emprendimientos, de qué tipo y en qué situación legal puedan encontrarse, sino que resulte difícil poder tener una idea de su estructura por tamaño o entidad. Eso sí, parece existir la posibilidad de obtener esa información de manera particular en las Cámaras de Comercio (www.confecamaras.org.co/index.php/directorio-de-camaras), pero no de manera integrada. La otra opción es la sugerida aquí, promediando los datos de las fuentes de información, a partir de indicadores indirectos, como puede ser el Índice de Densidad Industrial producido por el DANE y las informaciones ofrecidas puntualmente, en notas de prensa, informes o estudios, sobre emprendimientos nacionales y su dinámica. 


\section{Cuadro 1. Magnitudes del Cooperativismo en Colombia, 2003-2010.}

\begin{tabular}{|c|c|c|c|}
\hline & 2003 & 2010 & $\operatorname{TCMAA}^{(7)}$ \\
\hline Cooperativas $^{(1)}[\mathrm{a}]$ & 5.107 & 8.533 & 7,6 \\
\hline Entidades Totales activas en Colombia ${ }^{(2)}[\mathrm{b}]$ & 1.325 .276 & 2.267 .190 & 8,0 \\
\hline Sociedades en Colombia $(3)$ & 934.620 & 1.138 .915 & 2,9 \\
\hline$[\mathrm{a}] /[\mathrm{b}]$ en $\%$ & 0,4 & 0,4 & \\
\hline Asalariados en Cooperativas ${ }^{(1)}[c]$ & 80.987 & 139.703 & 8,1 \\
\hline Asalariados Totales $^{(4)}[\mathrm{d}]$ & 8.497 .983 & 9.750 .474 & 2,0 \\
\hline$[c] /[d]$ en $\%$ & 1,0 & 1,4 & \\
\hline Ocupados en Cooperativas ${ }^{(1)}[e]$ & 266.820 & 537.232 & 10,5 \\
\hline Ocupados Totales ${ }^{(4)}[\mathrm{f}]$ & 15.675 .381 & 18.129 .156 & 2,1 \\
\hline$[e] /[f]$ en $\%$ & 1,7 & 3,0 & \\
\hline VAB de las Cooperativas ${ }^{(1)(9)}[g]$ & 2.076 .236 & 8.554 .921 & 16,9 \\
\hline $\operatorname{VAB} \operatorname{Total}^{(5)(9)}[\mathrm{h}]$ & 249.155 .000 & 489.236 .000 & 4,6 \\
\hline$[\mathrm{g}] /[\mathrm{h}]$ en $\%$ & 0,8 & 1,7 & \\
\hline Remuneración de asalariados de las Cooperativas ${ }^{(1)(9)}[i]$ & 1.368 .896 & 6.466 .380 & 19,3 \\
\hline Remuneración de asalariados Total(5)(9) [j] & 77.610 .000 & 151.115 .000 & 4,5 \\
\hline$[\mathrm{i}] /[\mathrm{j}]$ en $\%$ & 1,8 & 4,3 & \\
\hline Transferencias sociales en especie de las cooperativas ${ }^{(6)(9)}[k]$ & 170.346 & 524.560 & 11,9 \\
\hline Transferencias sociales en especie Totales ${ }^{(5)(9)}[\mathrm{l}]$ & 8.351 .000 & 19.528 .000 & 7,4 \\
\hline$[k] /[1](8)$ en $\%$ & 2,0 & 2,6 & \\
\hline
\end{tabular}

FUENTES Y NOTAS: (1) Informe CENICOOP-2013 (Alarcón, 2013). (2) CONFECÁMARAS y Encuesta Anual Manufacturera del DANE. (3) CONFECÁMARAS, Informes anuales de gestión, Boletines y Noticias en www.confecamaras.org.co. (4) Encuesta Continua de Hogares y Gran Encuesta Integrada de los Hogares, para las secciones de Fuerza Laboral, Seguridad Social y Sexo. (5) Cuentas Nacionales Anuales del DANE. (6) Estimación como producto de los asalariados de las cooperativas por el diferencial salarial por rama productiva, para las 10 ramas finalmente homogeneizadas, entre el obtenido por la economía nacional respecto al remunerado por entidades cooperativas. (7) Tasa de Crecimiento Media Anual Acumulativa 2003-2010. Para el VAB, Remuneración de Asalariados y Transferencias Sociales en Especie se resta la inflación media anual acumulativa, un 5,5 por 100, según las series de Índices de Precios al Consumidor del DANE. (8) Relativamente a las Transferencias Sociales en Especie Nacionales junto a las estimativas de las cooperativas, de forma que el indicador termine correspondiendo a una proporción como parte del total. (9) Millones de pesos colombianos.

ping que podría afectar a los tratados de libre comercio y por ello se genera una supervisión más estricta de este tipo de entidades y muchas de estas se liquidan. Claro está que no todas las CTA existentes podrían enmarcarse dentro de esta fase instrumental, pero las existentes no lograron, siguiendo el análisis cualitativo sobre la Teoría Fundamentada de Cuñat (2015), generar sinergias en red que les permitiera aprender de las prácticas indebidas y diferenciar sus prácticas a través de la gestión reputacional que anclara en el mercado su legitimidad. 


\section{LA DIMENSIÓN SECTORIAL DEL COOPERATIVISMO COLOMBIANO A TRAVÉS DE UN ANÁLISIS DE REDES}

Así, la expansión de las cooperativas, efectivamente, se dio en un contexto de crecimiento nacional, pero de particular asimilación de las cooperativas a las sociedades tradicionales capitalistas y, con ello, la opción de un sistema para "cooperativizar" la economía se termina abandonando en pro de un proyecto de tercerización laboral y al unísono la promulgación de leyes que terminaron asimilando a las cooperativas del sector financiero a sus pares capitalistas y enfatizando la competencia por los mercados.

Al respecto de la clasificación sectorial que se utilizará en los cuadros y gráficos de este trabajo, se basará en la intersección de la sectorización entre las distintas fuentes de datos consultadas, siempre sujeta a la homogeneidad que requieren los indicadores, en especial los relativos al empleo asalariado y total, que son necesarios para la construcción y comparabilidad de muchas de las magnitudes utilizadas. Así, serán 10 sectores que resumen la actividad productiva de las cooperativas colombianas y de la economía de Colombia, siempre a partir de la Clasificación Internacional Industrial Uniforme Revisión 3 (Esquema 1).

La desagregación ha resultado ser más manejable (en cuadros, gráficos y líneas de texto) para su estudio, al sintetizar de manera sencilla la economía en una decena de sectores, evitando problemas de interpretación de las magnitudes obtenidas de las Cuentas Satélite para sectores de poca 0 mínima importancia cooperativa, así como la de cualquier relativización sobre el mismo sector nacional. En caso contrario no se atentaría a la congruencia de la suma de las partes con respecto al total, e incomodaba las interpretaciones para las comparaciones en el tiempo, es decir, el crecimiento de magnitudes sectoriales, y en el espacio, en referencia a las magnitudes de la economía colombiana.

\section{Esquema 1. Agregación de los Sectores de la Clasificación Internacional Industrial Uniforme, en su Revisión 3, en sectores homogeneizados para la comparabilidad de las variables de interés}

\begin{tabular}{|ll|c|}
\hline \multicolumn{2}{|c|}{} & CIIU Rev.3 \\
\hline 1 & AGRICULTURA, GANADERIÁ, CAZA, SILVICULTURA Y PESCA & $01-05$ \\
2 & EXPLOTACION DE MINAS Y CANTERAS & $06-09$ \\
3 & INDUSTRIAS MANUFACTURERAS & $10-37$ \\
4 & SUMINISTROS DE ELECTRICIDAD, GAS Y AGUA & $38-40$ \\
5 & CONSTRUCCION & $41-42$ \\
6 & COMERCIO AL POR MAYOR Y AL POR MENOR; REPARACIONES & $43-45$ \\
7 & TRANSPORTE, ALMACENAMIENTO Y COMUNICACIONES & $46-50$ \\
8 & INTERMEDIACION FINANCIERA & 51 \\
9 & ACTIVIDADES INMOBILIARIAS, EMPRESARIALES Y DE ALQUILER & $52-53$ \\
10 & EDUCACION, SALUD, SERVICIOS SOCIALES Y OTRAS ACTIVIDADES DE SERVICIOS & $54-61$ \\
\hline
\end{tabular}

FUENTE: Elaboración propia. 
En consecuencia, se supondrá que los sectores son homogéneos, y que el sentido matemático es el económico en la agregación, facilitando la cuestión de la comparabilidad. Esto requiere especial atención en el tratamiento de las Matrices Input-Output, pues cualquier comparación en el tiempo de una variable del cooperativismo respecto a la economía nacional, o del peso específico en cierta rama productiva de la economía nacional, queda fuera de toda posible comparación sin un mínimo común sectorial.

Así las cosas, se muestra una importancia considerable del sector cooperativo de la economía de Colombia, que alcanza cifras relativas sectoriales muy similares en términos de empleo total 0 entidades a lo que supone, por ejemplo, la rama de Explotación de minas y canteras nacional en el VAB colombiano -1,2 por 100 de entidades y 1,1 del empleo-, la mitad del VAB generado por el sector de Suministros Energéticos o la Intermediación Financiera nacionales -3,5 0 5,1 por 100 del VAB colombiano-, 0 a las de los asalariados del sector de la Construcción -5 por 100 de los asalariados colombianos- (Cuadro 2). Aspectos que son causa (o consecuencia técnica) de las especializaciones cooperativas tanto en producción, como en entidades y empleo, en los sectores de servicios: Intermediación Financiera, Educación, Salud y Servicios Sociales que, además, ven aumentada su especialización.

\section{Configuración de la Economía Cooperativa de Colombia según el Análisis de Redes Sociales ${ }^{8}$}

Conviene comenzar con un concepto básico en el sucesivo tratamiento de las redes de interrelaciones de sectores productivos de una economía o un segmento de ella (cooperativismo). Así, un grafo es un par ordenado "(V,E)" en el que "V" es un conjunto no vacío de nodos y "E" es un conjunto de enlaces entre nodos. Donde " $V$ " consta de pares no ordenados de nodos, tales que " $\{x, y\}$ " pertenecen a "E" y se dice que " $x$ " e "y" son adyacentes. En su expresión gráfica, se representa mediante líneas que unen dichos nodos, y una flecha orientada si el grafo es dirigido, pudiendo ir de un sentido a otro, 0 a ambos, entre " $x$ " $e$ " $y$ ".

Así pues, la consistencia y vertebración de la economía nacional o de su segmento cooperativo a partir de las sus relaciones intersectoriales, puede introducirse si se parte de la idea de centralidad del grafo de tales relaciones. El término tiene sus orígenes en el concepto sociométrico de red estrella, que matemáticamente tiene el máximo grado de centralización. Así, un nodo (sector productivo) adquiere el protagonismo de las relaciones en su conjunto si se relaciona con todos los demás, y su formalización tiene un importante precedente en los trabajos, pioneros en la materia, que realizó Bavelas (1948) en los años 40 y 50.

8.- Los algoritmos y la notación formal original de los indicadores sobre Análisis de Redes Sociales que se exponen a continuación se encuentran en las referencias y en la ayuda del software UCINET 6 de Analytic Technologies. Este software, ingeniado por Borgatti, Everett y Freeman (2002) se encuentra en http://www.analytictech.com/. 


\section{Cuadro 2. Especialización sectorial(1) del VAB, entidades, asalariados y ocupados (Empleo) de la economía cooperativa de Colombia sobre la nacional en 2003 y 2010}

\begin{tabular}{|c|c|c|c|c|c|c|c|c|}
\hline \multirow{2}{*}{$\begin{array}{l}\text { Sectores } \\
\text { (2) }\end{array}$} & \multicolumn{4}{|c|}{2003} & \multicolumn{4}{|c|}{2010} \\
\hline & VAB & Entidad & Asalariad & Empleo & VAB & Entidad & Asalariad & Empleo \\
\hline 1 & 0,18 & 1,02 & 0,10 & 0,22 & 0,61 & 1,72 & 0,03 & 0,22 \\
\hline 2 & - & 0,50 & 0,18 & 0,10 & 0,09 & 0,50 & - & 0,36 \\
\hline 3 & 0,47 & 0,15 & 0,59 & 0,30 & 0,25 & 0,16 & 0,64 & 0,33 \\
\hline 4 & - & 1,33 & 0,14 & 1,00 & 0,09 & 0,50 & - & 0,40 \\
\hline 5 & 0,15 & 0,49 & 0,20 & 0,24 & 0,08 & 0,33 & 0,04 & 0,35 \\
\hline 6 & 1,19 & 0,47 & 1,27 & 0,35 & 0,88 & 0,35 & 1,10 & 0,25 \\
\hline 7 & 0,68 & 1,59 & 2,62 & 0,92 & 0,46 & 1,34 & 1,51 & 0,49 \\
\hline 8 & 5,86 & 8,06 & 8,05 & 4,25 & 3,29 & 7,39 & 15,64 & 7,69 \\
\hline 9 & 0,37 & 0,26 & 1,91 & 3,58 & 0,28 & 0,23 & 0,46 & 1,44 \\
\hline 10 & 4,26 & 3,79 & 0,84 & 2,45 & 6,73 & 4,23 & 0,73 & 3,04 \\
\hline
\end{tabular}

NOTAS: (1) El coeficiente de especialización cooperativa de cada Variable en cada rama productiva "i" es

$$
\beta i=\frac{\frac{\text { Variable Cooperativa }_{i}}{\sum_{i} \text { Variable Cooperativa }_{i}}}{\frac{\text { Variable Nacional }_{i}}{\sum_{i} \text { Variable Nacional }_{i}}}
$$

De tal manera que si $\beta i>1$ el cooperativismo está especializado relativamente para la variable referida en el sector productivo "i" respecto a la media nacional, y viceversa si ßi<1. (2) Los 10 sectores productivos se corresponden con la clasificación del Esquema 1.

FUENTE: Informe CENICOOP-2013 (Alarcón, 2013).

Por otra parte, Freeman (1979) distingue la "centralidad de los nodos" de la "centralidad del grafo" debido a una mayor densidad de nodos protagonistas en la comparación de los grafos, para distinguir su respectivo poder en el conjunto. De manera que la propuesta de Scott (1991), para evitar esa confusión terminológica, consiste en reservar el término "centralidad" para la cuestión de la centralidad de los puntos y utilizar el de "centralización" para referirse al problema de la cohesión interna del grafo tomado como un todo. En consecuencia, este trabajo seguirá a Freeman (1979) en las formas de cálculo de la centralidad (Hanneman y Riddle, 2005). Así, la medida más simple e intuitiva de centralidad es a través del grado $\left(\mathrm{k}_{\mathrm{i}}\right)$ o número de relaciones de los "i" nodos -sectores-, es decir, de la red de relaciones intersectoriales. Se notaría como 


$$
\mathbf{k}_{\mathbf{i}}=\sum_{\mathrm{j}} \mathrm{a}_{\mathrm{ij}}
$$

siendo "aij" los elementos de la matriz de adyacencia "A", cuyas celdas son 1 ó 0 según exista una relación intersectorial significativa o no.

Así, un grafo dirigido dispone de grado de entrada al nodo (columna) o sector productivo y de salida de él (fila). De manera que un sector productivo es central conforme tiene mayor grado, lo que se corresponde con la idea intuitiva según la cual un sector está bien conectado con los demás de su entorno.

A parte de ese aspecto local en la red de relaciones intersectoriales, es muy interesante introducir la idea de intermediación del sector "k", que para Freeman (1979) determina la medida en la que una rama " $k$ " hace de intermediaria entre otros sectores por estar situado en el camino de estos, y se notaría como

$$
\mathbf{b}_{\mathbf{k}}=\Sigma_{\mathrm{i}} \Sigma_{\mathrm{j}} g_{\mathrm{ikj}} / g_{\mathrm{ij}}
$$

donde, y "j".

$g_{i k j}=$ número de veces que aparece el sector productivo "k", en los caminos entre los sectores "i" $g_{i j}=$ total de caminos posibles entre "i" y "j".

Una derivación de la misma idea, pero que incluye las relaciones indirectas de intermediación, es decir, no sólo las distancias geodésicas directas -óptima o mínimas entre nodos-, sino también caminos indirectos para la intermediación entre dos sectores es la centralidad del flujo de intermediación? Dada una matriz de adyacencia ${ }^{10}$ de "j x k" nodos-sectores, siendo " $m_{j k}$ " el flujo entre el sector "j" y el sector " $k$ " que debe de pasar por el sector "i" para cualquier camino (máximo). El flujo de intermediación del sector "ii" es la suma de todos los " $m_{j k}$ " donde "i", "j" y "k" son distintos y "j < k". La centralidad del flujo de intermediación será una medida de la contribución de un sector productivo a todos los flujos máximos posibles.

A través del Análisis de Redes Sociales (ARS, en lo sucesivo) también puede estudiar hasta qué punto la red de relaciones intersectoriales se constituye, o no, como una estructura centralizada. Para ello se ayuda de tres indicadores: la densidad, es decir, la relación porcentual del número de relaciones entre el total de las posibles; la cohesión, definida en teoría de redes como el esfuerzo de rela-

9.- Se obtiene como diferencia relativa entre el máximo flujo entre cualesquiera nodos de la red, a través de la matriz $W$, y el resultante de eliminar la matriz W la fila y columna " $k$ " referida al nodo o sector productivo del que se pretende obtener el indicador del flujo de intermediación, a la que se llamará $W^{-k}$. Notando en minúscula los elementos de tales matrices se formalizaría el flujo de intermediación de " $k$ " como $f b_{k}=\sum_{i j}\left(w_{i j}-w^{-k} \cdot{ }_{i j}\right) / w^{-k}$

10.- Matriz binaria, en la que "0" señala que no hay vínculo entre sectores productivos, y "1" muestra que sí lo hay. 


\section{LA DIMENSIÓN SECTORIAL DEL COOPERATIVISMO COLOMBIANO A TRAVÉS DE UN ANÁLISIS DE REDES}

cionarse entre ramas productivas, que determina el número de relaciones en el camino más corto entre ambos; y el grado de centralización -Network Centralization Index, más conocido por su siglas en inglés como NCl, en lo sucesivo)-, que es la variabilidad en grados como porcentaje sobre la máxima centralización -es decir, en relación a la red estrella como estructura del máximo poder de un solo sector productivo sobre el conjunto-. Hay diferentes indicadores según la concepción de centralidad de los nodos a tratar (Borgatti, Everett y Freeman, 2002).

En este trabajo se utilizarán dos medidas de centralidad interpretables por parte de marco InputOutput: el grado y la centralidad del flujo del nodo en las versiones "desde" y "hasta" el sector que se trate. A estas se añaden las medidas de densidad, cohesión, su complementario, la fragmentación, y el índice de centralidad de la red, para las dos medidas sobre los nodos-sectores, como indicadoras de la consistencia o vertebración de las relaciones intersectoriales conjuntas que se van a tratar.

Por último, la fragmentación es la proporción de pares de nodos-sectores que no pueden alcanzar al resto. Es una medida casi complementaria de la cualidad de compactación de una red (cohesión), que parte del número de enlaces que pueden alcanzar a otros nodos de la red. Como la distancia entre dos sectores productivos se considerará a la longitud del camino más corto, y la distancia genérica será la longitud de un camino óptimo se tiene que, en una matriz de adyacencia, la distancia y la distancia genérica serán equivalentes, de manera que la matriz de cercanía se confecciona por medio de una transformación que calcula los caminos más cortos entre los posibles caminos.

La interpretación de la dimensión sectorial del cooperativismo que se pretende aquí va a ayudarse de la obra clásica de Clark (1980) en los años 40: Conditions of Economic Progress, y de su artículo de 1949 (Clark, 1949), donde se expone una taxonomía de los sectores productivos en función de sus interrelaciones. Además, desde el marco Input-Output puede interpretarse fácilmente esa taxonomía, así se podrá caracterizar la configuración productiva intersectorial de segmento económico cooperativo, para compararla después con la correspondiente a la Economía Nacional.

La taxonomía de los sectores productivos se populariza en el ámbito de la Economía Aplicada con los coeficientes de Rasmussen (1956) en los años 50, y en el trabajo no requiere de cambios sustanciales, conscientes de las distintas sectorizaciones desde entonces, debidas a los avances en la disponibilidad de información y en la estadística producidos, así como en los cambios de concepciones sectoriales que se han producido desde entonces. Así, el autor viene a tratar los aspectos sobre los arrastres hacia delante y hacia atrás de los sectores productivos, referidos a sus relaciones con respecto a los demás. Y eso no es otra cosa que categorizar tales sectores de manera muy cercana a como lo hacen clasificaciones basadas en los efectos multiplicadores I-O sectoriales originarios, pues los coeficientes de las columnas de la matriz $(\mathrm{I}-\mathrm{A})^{-1}$-siendo $\mathrm{A}$ la matriz de coeficientes interiores- indican la cuantía en que debe aumentar la producción de un sector i-ésimo para que la demanda final de un sector j-ésimo se incremente en una unidad. 
De la matriz inversa de Leontief, por tanto, se pueden obtener los coeficientes que recojan la capacidad de los distintos sectores de la Economía o del cooperativismo de generar o de absorber crecimiento. De esa manera, las sumas de los elementos de cada fila y columna de dicha matriz, esto es, los denominados efectos absorción $\left(\alpha_{i}\right.$. ) y difusión $\left(\alpha_{. j}\right)$, respectivamente, se obtienen calculando un promedio de dichos efectos en cada una de las ramas, para después expresar cada uno de estos promedios en relación a los efectos globales producidos. Sobre tales conceptos, y bajo los criterios de Clark y Rasmussen, la clasificación de las ramas productivas distingue entre:

a) Los sectores Clave de una Economía, que son aquellos que presentan unos efectos de arrastre hacia adelante y hacia atrás superiores a la media;

b) los sectores con efectos de arrastre hacia atrás mayores que la media y adelante menores que la media, denominados Motores;

c) sectores con eslabonamientos hacia adelante sobre la media, y hacia atrás por debajo, denominados sectores o ramas Base;

d) y los sectores Independientes, que presentan unos efectos de dispersión y absorción por debajo de la media.

Teniendo en cuenta esta clasificación (Cuadro 3), hay importantes diferencias estructurales entre la economía colombiana su segmento cooperativo, si bien conviene decir que los sectores clasificados cambian, pero no genérica ni sustancialmente en la etapa. El sector Agrario se consideraría Independiente para la economía colombiana, mientras que es Clave para el subsistema cooperativo en 2003, y Motor cooperativo entre 2004 y 2010. La Energía sería una rama Motor para la economía nacional e Independiente para las cooperativas -entidades que pesan poco en un sector característico de grandes empresas intensivas en capital físico-. La Construcción es Motor en ambas, y la rama Inmobiliaria y servicios empresariales es Independiente para el sistema cooperativo y Base para la economía nacional, es decir, con ciertos arrastres hacia delante "netos" (Cuadros 3 y 4). Por lo demás, los sectores Motores nacionales son la Manufactura, Construcción, Comercio y hostelería y Transporte y comunicaciones.

De esas ramas Motores se intuye cierta adaptación del sector cooperativo a la configuración nacional, pues en las cuatro ramas nacionales persiste el peso cooperativo e incluso aumenta, especialmente en el caso del Comercio y hostelería, rama en la que el cooperativismo está especializado. La Minería es una rama de poco peso cooperativo (0,8 por 100) y aún menor respecto a la Minería de la economía nacional (0,17 por 100), que tiende a la Independencia desde un inicio Motor en 2003.

Caso aparte es el de las cooperativas de crédito, en tanto que comparten la tendencia a la Independencia con la economía nacional en 2010, cuando en 2003 eran una rama Motor, mientras que para el cooperativismo eran rama Base, es decir, con efectos netos hacia delante. Una dinámica derivada, probablemente, por su elevado peso relativo: 1 de cada 5 unidades monetarias generadas del valor añadido cooperativo y casi un 6 por 100 del VAB de la Intermediación Financiera nacional en 2010. 


\section{Cuadro 3. Caracterización sectorial de Clark-Rasmussen para la economía cooperativa y la nacional de Colombia en 2003 y 2010}

\begin{tabular}{|l|c|c|c|c|}
\hline Sectores $(1)$ & \multicolumn{2}{|c|}{2003} & \multicolumn{2}{c|}{2010} \\
& Economía Cooperativa & Economía Nacional & Economía Cooperativa & Economía Nacional \\
\hline 1 & CLAVE & INDEPEND. & MOTOR & INDEPEND. \\
2 & MOTOR & INDEPEND. & INDEPEND. & INDEPEND. \\
3 & CLAVE & CLAVE & MOTOR & CLAVE \\
4 & MOTOR & MOTOR & INDEPEND. & MOTOR \\
5 & MOTOR & MOTOR & MOTOR & MOTOR \\
6 & MOTOR & MOTOR & MOTOR & MOTOR \\
7 & MOTOR & MOTOR & MOTOR & MOTOR \\
8 & BASE & MOTOR & INDEPEND. & INDEPEND. \\
9 & INDEPEND. & BASE & INDEPEND. & BASE \\
10 & INDEPEND. & INDEPEND. & INDEPEND. & INDEPEND. \\
\hline
\end{tabular}

NOTAS: (1) Los 10 sectores productivos se corresponden con la clasificación del Esquema 1.

FUENTE: Informe CENICOOP-2013 (Alarcón, 2013).

También cabe referirse a que las ramas de mayor peso cooperativo, la de Intermediación Financiera y la de Educación Salud y otros servicios personales y colectivos, no se caracterizan como motores, y sí lo hacen los sectores clásicos, agricultura y manufactura, fundamentalmente, como ocurre en el caso de la economía nacional. Todo ello es reflejo, en parte, de la dinámica de los multiplicadores cooperativos hacia atrás, los cuales son, como media, menores que los nacionales, al contrario que los multiplicadores cooperativos hacia delante.

En cuanto a los multiplicadores del empleo (Cuadro 4), son mayores para el cooperativismo que para la economía nacional en 2010 -salvo en los sectores no manufactureros, ni extractivos, ni de servicios, y en el de Educación, Salud, y otros servicios personales y colectivos-, lo cual explica la mayor intensidad del factor trabajo cooperativo en esos sectores, y que ante caídas de la demanda se pueda destruir relativamente más empleo. No obstante, tales multiplicadores experimentan un cambio notable en la etapa que ocupa el trabajo, de manera que los correspondientes a la economía nacional en 2003 eran mayores que los del segmento cooperativo -salvo para las ramas de Minería, Suministros energéticos, electricidad y agua y Actividades Inmobiliarias y servicios a empresas-. Además, esos multiplicadores del empleo cooperativo no se han visto reducidos en la misma medida que los correspondientes a la economía nacional y, por tanto, es posible afirmar que las cooperativas en su conjunto 


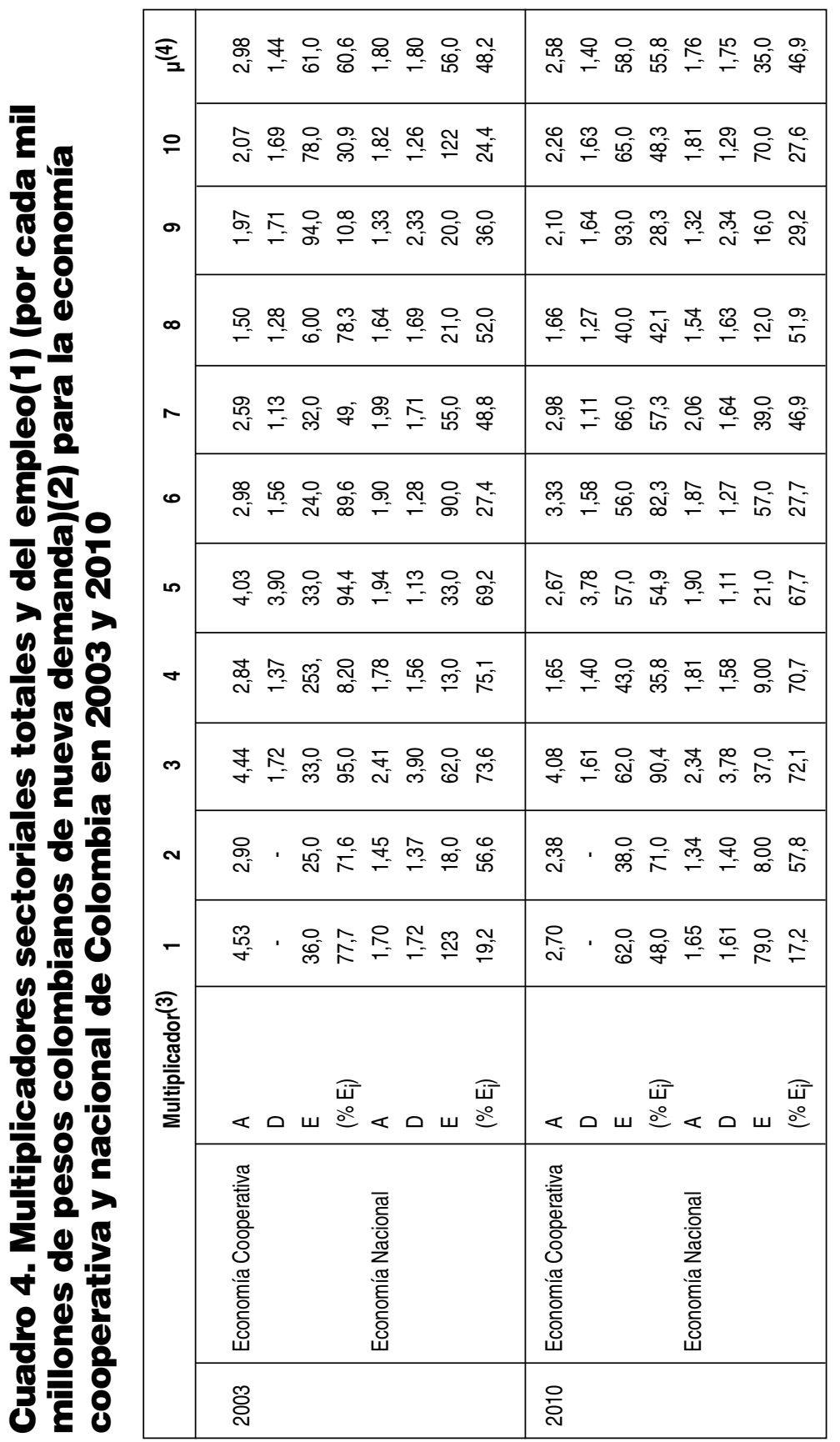

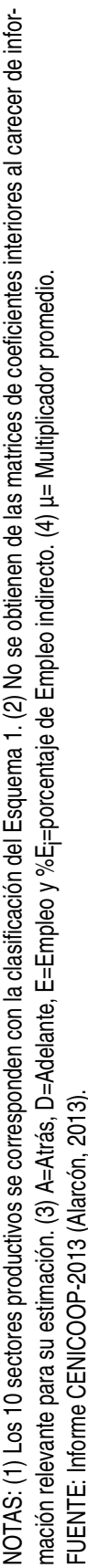




\section{LA DIMENSIÓN SECTORIAL DEL COOPERATIVISMO COLOMBIANO A TRAVÉS DE UN ANÁLISIS DE REDES}

resisten mejor las dificultades de las fases contractivas, tal como lo demuestra Sala et al. (2014), aunque las CTA colombianas parecen seguir los hallazgos que para las sociedades laborales españolas reportan estos autores, es decir, que en las fases expansivas son las CTA las que han aumentado a mayor ritmo, pero en las de recesión tienden a perder más empleo que el sector cooperativo en su conjunto. Quizá se cumpla alguna de las hipótesis de Navarra (2016) al respecto de que CTA operen con un margen de flexibilidad en los salarios o los mantengan bajos sobre la media para acumular capital y poder enfrentarse con mayor solidez a ciclos recesivos y estabilidad en el empleo. En todo caso, los multiplicadores totales -los técnicos-, que vienen a explicar los del empleo, muestran también una caída, tanto en el caso cooperativo como nacional de la economía, y en sus efectos atrás como adelante.

La distinta caracterización sectorial del mundo cooperativo respecto a la economía nacional se conforma de nuevo con la mayor generación de empleo indirecto, salvo en los sectores de Suministros energéticos, Construcción, Comercio y hostelería e Intermediación Financiera (Cuadro 4). Así, a lo largo del periodo, los multiplicadores sectoriales del empleo nacional caen, y los cooperativos aumentan, salvo para las ramas de Suministros de electricidad, gas y agua, Inmobiliarias y servicios a las empresas y Educación, salud y otros servicios personales o colectivos, que eran más intensivos en mano de obra al inicio, aspecto que destaca cierto proceso de capitalización cooperativa -introducción de más capital físico-, posiblemente asociado a la introducción de mejoras tecnológicas.

Así que, en 2003, esos sectores tienen multiplicadores del empleo por encima de la media, y en 2010 se añaden a ellos los sectores Agrario, la Manufactura y el de Transporte y comunicaciones (Cuadro 4), saliendo de la lista la rama de Suministros de electricidad, gas y agua. En consecuencia, se va adquiriendo una configuración cooperativa similar a la de la economía nacional en 2010, en cuanto a sectores con multiplicadores del empleo sobre el promedio: Agrario, Manufactura y Comercio y hostelería.

\section{Configuración de la red de relaciones intersectoriales de las cooperativas en Colombia y su dinámica reciente}

La utilización instrumental de la teoría de grafos hace posible visualizar de manera inmediata las diferencias entre redes de relaciones intersectoriales configuradas por la Economía Nacional y la Economía Cooperativa, así como determinar el protagonismo de los sectores o ramas productivas en la estructura de la red por medio de medidas de centralidad, entre otras. Tales indicadores se interpretan como resúmenes de la influencia e importancia de estos sectores en la configuración nacional de las transacciones intersectoriales bajo un enfoque diferente, que bien puede enriquecer las interpretaciones convencionales. En este apartado se presentarán los cálculos de tales indicadores para observar ciertas cualidades de los sectores productivos colombianos y los de su cooperativismo, así 
como otros sobre el conjunto de la red, que son los referidos a la centralización del grafo, también en ambos casos (Borgatti, Everett y Freeman, 2002; Alarcón, 2009, 2016).

La posición intermedia de una rama productiva en las relaciones económicas entre otros sectores significa que la misma puede tener algún control sobre las transacciones de las ramas no adyacentes, es decir, no relacionadas directamente, y por eso conviene incluir en el algoritmo de solución otros caminos que pongan en contacto a esos dos nodos- sectores. Así, este indicador refleja el poder de la rama productiva "mayormente intermediaria", tanto para desparramar sus efectos beneficiosos como los perjudiciales. Por lo que caminos alternativos provocarán una menor dependencia de ese sector para desarrollar las actividades económicas y, por tanto, la disminución del poder del intermediador. De ahí la dual interpretación. Piénsese, como un buen ejemplo de una rama productiva de poder intermediador, en el caso de la Intermediación Financiera, valga la redundancia del significante, y las consecuencias a las que pueden llevar -o han llevado- sus aristas en el desenvolvimiento económico ante dinámicas recesivas. De manera que lo anterior tiene cierto reflejo en el trabajo de Ndiege et. al (2016), que estudia un sistema financiero diferente al colombiano (Tanzania), en el que las entidades financieras de la economía social son jóvenes y parten de niveles de eficiencia bajos (estudio sobre 60 entidades), pero el persistente auge y captación de cuotas de ahorro e inversión y ganancia de eficiencia es consecuencia de las redes de colaboración entre ellas. De tal manera que, si son aprovechadas las economías de escala de la cooperación y colaboración en la asunción conjunta de costes de expertos y de tecnología, continuará la convergencia en eficiencia y de su influencia en la economía.

Los conceptos e indicadores anteriores aplicados implican que, en los dos sentidos de la centralidad-como son las capacidades de una rama de ser proveedora o receptora dentro de las relaciones productivas intersectoriales, es decir, los Grados de Salida o Entrada- las estructuras sectoriales de las cooperativas colombianas y de su economía nacional difieren notablemente. En efecto, a través del grado de centralidad de Freeman (1979) vuelve a destacarse lo que de la configuración de la red cooperativa y la de la Economía Cooperativa se derivaba en el modelo Input-Output. Así, el sector Agrario, las ramas de Manufactura, y la Intermediación Financiera persisten en los primeros puestos de centralidad, y también como sectores que arrastran al resto en el caso de las cooperativas, mientras que para la economía nacional se añaden el sector Inmobiliario y de servicios a empresas y el de Transportes y comunicaciones, para ejercer ese rol, sustrayendo del conjunto a la Intermediación Financiera. En cuanto a los sectores que se ven arrastrados, el cooperativismo enumera al Minero, Construcción, Comercio y hostelería y Educación, Salud, y otros servicios personales y colectivos (Cuadro 5 y Gráficos 1 a 4).

Por otra parte, en cuanto a la concentración de la red -centralización como indicador conjunto-, los indicadores obtenidos destacan que, inicialmente, la estructura de las relaciones intersectoriales está más centralizada para la economía cooperativa que para la nacional. El grado medio tiende a igualarse entre las redes partiendo desde el ligeramente mayor de la economía nacional (Cuadro 6). 


\section{LA DIMENSIÓN SECTORIAL DEL COOPERATIVISMO COLOMBIANO A TRAVÉS DE UN ANÁLISIS DE REDES}

Las estructuras pierden centralidad respecto a los sectores señalados en el párrafo anterior, como claramente manifiesta el poder de centralidad de la Manufacturas en la economía nacional, rama en la cual toma posiciones también el cooperativismo. Y destaca el peso de la Intermediación Financiera cooperativa, en un diferencial similar al papel del sector de Transporte y comunicaciones o la rama de Actividades Inmobiliarias y servicios a empresas para la economía nacional. Todo ello sin olvidar al sector Agrario, que es el siguiente en el orden de centralidad en ambas redes de relaciones intersectoriales, sobre el que se percibe el cambio estructural del sector, perdiendo centralidad en la economía nacional (Cuadro 5 y Gráficos 1 a 4). En definitiva, que las características motoras de ciertas ramas del cooperativismo colombiano redundan en su carácter más central.

\section{Cuadro 5. Centralidad del Grado de Entrada (GE) y de Salida (GS) de los sectores productivos en la red cooperativa y nacional de la economía de Colombia y centralización de ambas redes, en 2003 y 2010}

\begin{tabular}{|c|c|c|c|c|c|c|c|c|}
\hline \multirow[t]{3}{*}{ Sectores $(1)$} & \multicolumn{4}{|c|}{ ECONOMIA COOPERATIVA } & \multicolumn{4}{|c|}{ ECONOMIA NACIONAL } \\
\hline & \multicolumn{2}{|c|}{2003} & \multicolumn{2}{|c|}{2010} & \multicolumn{2}{|c|}{2003} & \multicolumn{2}{|c|}{2010} \\
\hline & GS & GE & GS & GE & GS & GE & GS & GE \\
\hline 1 & 4 & 3 & 3 & 3 & 3 & 0 & 3 & 2 \\
\hline 2 & 0 & 3 & 0 & 2 & 2 & 3 & 2 & 2 \\
\hline 3 & 8 & 3 & 8 & 4 & 9 & 0 & 8 & 4 \\
\hline 4 & 0 & 2 & 0 & 1 & 1 & 4 & 1 & 3 \\
\hline 5 & 0 & 4 & 0 & 2 & 0 & 3 & 0 & 2 \\
\hline 6 & 0 & 3 & 1 & 4 & 1 & 4 & 0 & 4 \\
\hline 7 & 1 & 2 & 0 & 3 & 4 & 5 & 2 & 4 \\
\hline 8 & 10 & 1 & 10 & 1 & 2 & 3 & 2 & 2 \\
\hline 9 & 1 & 1 & 2 & 2 & 8 & 0 & 8 & 1 \\
\hline 10 & 1 & 3 & 1 & 3 & 1 & 3 & 1 & 3 \\
\hline $\mathrm{NCl}^{*}$ & $95,83(2)$ & $26,39(3)$ & $95,83(2)$ & $26,39(3)$ & 79,17 & 23,61 & 70,33 & 29,17 \\
\hline Media NCl & \multicolumn{2}{|c|}{$61,11^{(4)}$} & \multicolumn{2}{|c|}{$61,11^{(4)}$} & \multicolumn{2}{|c|}{51,39} & \multicolumn{2}{|c|}{49,75} \\
\hline
\end{tabular}

NOTAS: (1) Los 10 sectores productivos se corresponden con la clasificación del Esquema 1. (2), (3) y (4) = El número igual de nodos implicados sobre un mismo total en los denominadores repercute en la igualdad de los indicadores calculados.

* Network Centralization Index

FUENTES: Informe CENICOOP-2013 (Alarcón, 2013) y Borgatti, Everett, y Freeman (2002). 
El cooperativismo colombiano va a participar también de la inercia del modelo de la economía nacional, como corrobora la centralidad de la Manufactura y de las Actividades Inmobiliarias y de servicios a empresas. Mientras tanto, las diferentes prácticas de asunción de riesgos financieros de las cooperativas de crédito mantienen a la rama de Intermediación Financiera en puestos de centralidad mayor que la rama nacional. Al respecto del sector de la Construcción, cabe decir que no aparece como destacado en la centralidad de la red intersectorial del sistema cooperativo presentado, según los indicadores propuestos (Cuadro 5; Gráficos 1 a 4).

En cuanto a la densidad de las relaciones intersectoriales (Cuadro 6), se observa un aumento relativo tanto en el sector cooperativo como en la economía colombiana hasta 2007, año en el que, con vaivenes, comienza una caída hasta 2010. Las relaciones intersectoriales cooperativas se hacen más densas, una vez que partían de niveles inferiores, ofreciendo una tendencia a la mayor cohesión, lo cual se confirma bajo los indicadores de compactación y fragmentación, con aumentos y caídas ligeras, respectivamente, y de manera contraria a la que ocurre con las redes de relaciones intersectoriales de la economía nacional. Con todo, a pesar de las tendencias a la convergencia, la fragmentación de las redes cooperativas es superior a la de las redes de la economía nacional, tanto como la compactación es menor. En consecuencia, no es concluyente que el cooperativismo constituya estructuras de mayor cohesión en las relaciones intersectoriales en las que se implique, puesto que el juego competitivo es una de las causas en la producción de mayores relaciones intersectoriales, y de la mayor vertebración o compactación (Cuadro 6). Es decir, que los grafos del cooperativismo se reflejan diferencialmente a los de la economía nacional -compuesta mayormente por entidades tradicionales capitalistas-, y que las cooperativas se adaptan a la producción sectorial nacional, debido también a su peso respecto a ella, la cual ha reducido su cohesión a lo largo de la etapa de estudio. Y lo ha hecho diferencialmente a la economía cooperativa. Habría que indagar si el tamaño del cooperativismo colombiano es suficiente como para que la inercia seguida en el alcance de la economía nacional termine ayudando 0 alejando en la breve contribución cooperativa al crecimiento económico colombiano, una vez que se sabe que es un sumando.

En definitiva, la representación de los grafos de las relaciones intersectoriales (Gráficos 1 a 4) señala, intuitivamente, que las configuraciones estructurales de las redes intersectoriales para el conjunto de la economía nacional y para el sector cooperativo difieren: grafos más densos y más cohesionados los de la economía nacional, más vertebrados. Los indicadores que evalúan al grafo como un todo confirman lo mismo: mayor cohesión y densidad de las redes de la economía nacional en comparación a las del sector cooperativo (Cuadro 6), pero las redes cooperativas intersectoriales tienden a cohesionarse más, mientras que las de la economía nacional tienden a fragmentarse más.

Por otra parte, también se deducen las capacidades de intermediación, es decir, de ser ramas con poder entre las relaciones intersectoriales indirectas de dos cualesquiera, en la rama cooperativa de la Manufactura, que es aún más intensa y protagonista en la red de la economía nacional respecto a la cooperativa, y en menor medida para el Comercio y hostelería, en similar diferencia de intensidad. Con todo, la diferencia fundamental entre las redes la protagoniza la rama de Transportes y comu- 


\section{Cuadro 6. Densidad y Cohesión de la red de relaciones intersectoriales cooperativa y nacional de la economía de Colombia entre 2003 y 2010}

\begin{tabular}{|l|c|c|c|c|c|}
\hline Sectores(1) & Año & Grado medio & Densidad & Fragmentación & Compactación \\
\hline \multirow{3}{*}{ Economía Cooperativa } & 2003 & 2,1 & 0,23 & 0,72 & 0,26 \\
& 2004 & 2,1 & 0,23 & 0,71 & 0,26 \\
& 2005 & 1,9 & 0,21 & 0,72 & 0,24 \\
& 2006 & 2,3 & 0,26 & 0,68 & 0,29 \\
& 2007 & 2,4 & 0,27 & 0,62 & 0,32 \\
& 2008 & 1,9 & 0,21 & 0,73 & 0,24 \\
& 2009 & 2,2 & 0,24 & 0,70 & 0,27 \\
& 2010 & 2,1 & 0,23 & 0,66 & 0,29 \\
\hline \multirow{3}{*}{ Economía Nacional } & 2003 & 2,3 & 0,26 & 0,57 & 0,34 \\
& 2004 & 2,4 & 0,27 & 0,37 & 0,42 \\
& 2005 & 2,2 & 0,24 & 0,54 & 0,34 \\
& 2006 & 2,2 & 0,24 & 0,54 & 0,34 \\
& 2007 & 2,2 & 0,24 & 0,54 & 0,35 \\
& 2008 & 2,0 & 0,22 & 0,60 & 0,31 \\
& 2009 & 2,2 & 0,24 & 0,50 & 0,36 \\
& 2010 & 1,9 & 0,21 & 0,63 & 0,29 \\
\hline
\end{tabular}

NOTAS: (1) Los 10 sectores productivos se corresponden con la clasificación del Esquema 1.

FUENTES: Informe CENICOOP-2013 (Alarcón, 2013) y Borgatti, Everett, y Freeman (2002).

nicaciones, con un elevado poder de intermediación en la red de relaciones intersectoriales de la economía nacional, y que se reduce de manera considerable en la etapa de estudio. Así, cabe también señalar que el mayor cambio que experimentan los grafos tiene que ver con esta rama, en consonancia con la revolución de las Tecnologías de Información y Comunicación, el cual queda visible en las redes de relaciones intersectoriales de la economía nacional, pero no en la cooperativa (Cuadro 7).

En definitiva, el sector cooperativo de la economía colombiana se adapta a la configuración económica nacional, y cambia en las partes en las que su peso lo permite, lo cual implica que la cohesión y la densidad de las redes de relaciones tratadas aumenten su magnitud en el ciclo, de tal manera que una mayor vertebración en tales relaciones no implica la capacidad de que la misma se constituya como un atributo positivo en el caso de un contexto recesivo, claro. 
Cuadro 7. Grado de intermediación sectorial de las redes cooperativa y nacional de la economía de Colombia 2003-2010

\begin{tabular}{|l|c|c|c|c|}
\hline \multirow{2}{*}{ Sectores (1) } & \multicolumn{2}{|c|}{ Economía Cooperativa } & \multicolumn{2}{c|}{ Economía Nacional } \\
& 2003 & 2010 & 003 & 2010 \\
\hline 1 & 0 & 0 & 3 & 0 \\
2 & 0 & 0 & 7 & 0 \\
3 & 4 & 9 & 0 & 13 \\
4 & 0 & 0 & 0 & 0 \\
5 & 0 & 0 & 0,5 & 0 \\
6 & 0 & 1 & 7,5 & 0 \\
7 & 0 & 0 & 0 & 1 \\
8 & 0 & 0 & 0 & 0 \\
9 & 0 & 0 & 0 & 0 \\
10 & 0 & 0 & 0 \\
\hline
\end{tabular}

NOTAS: (1) Los 10 sectores productivos se corresponden con la clasificación del Esquema 1.

FUENTES: Informe CENICOOP-2013 (Alarcón, 2013) y Borgatti, Everett, y Freeman (2002).

La razón es que la magnitud de los arrastres aplicados a configuraciones netamente motoras y centralizadas se aceleran en ciclos expansivos, pero se exponen a provocar un efecto dominó de serias repercusiones socioeconómicas en el caso contrario. Esto mismo parece confirmarse teniendo en cuenta los instrumentos de Economía Aplicada empleados: la menor vertebración del cooperativismo, relativamente a la economía nacional puede suponer un amortiguador en supuestos en la caída de la actividad económica. 
Gráfico 1. Grafo de la red de relaciones intersectoriales de la economía de Colombia en 2003

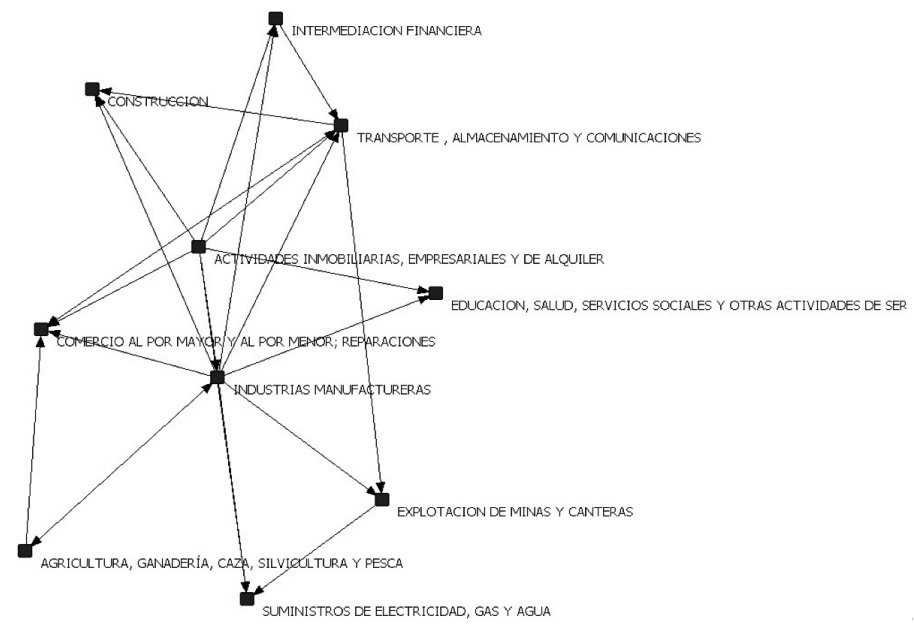

FUENTES: Informe CENICOOP-2013 (Alarcón, 2013) y Borgatti (2002).

\section{Gráfico 2. Grafo de la red de relaciones intersectoriales de la economía cooperativa de Colombia en 2003}

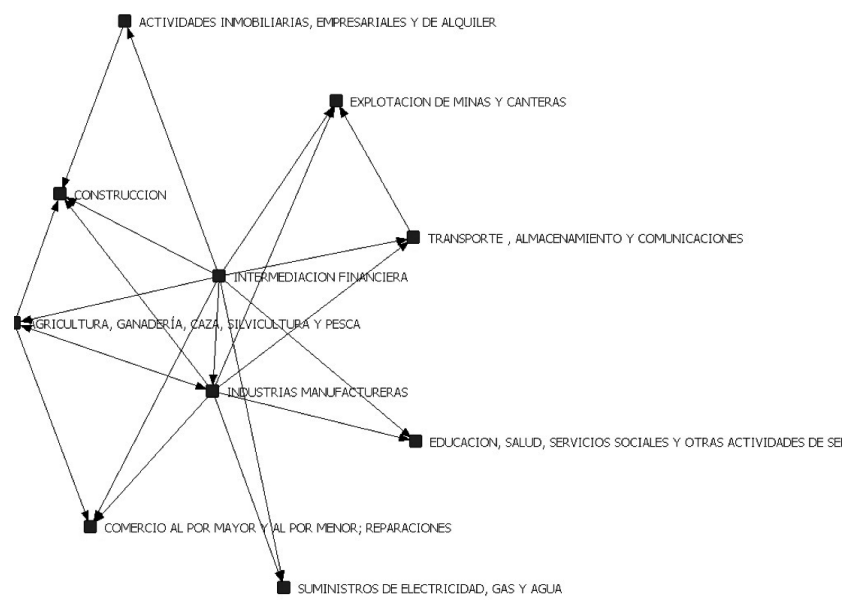

FUENTES: Informe CENICOOP-2013 (Alarcón, 2013) y Borgatti (2002). 


\section{Gráfico 3. Grafo de la red de relaciones intersectoriales de la economía de Colombia en 2010}

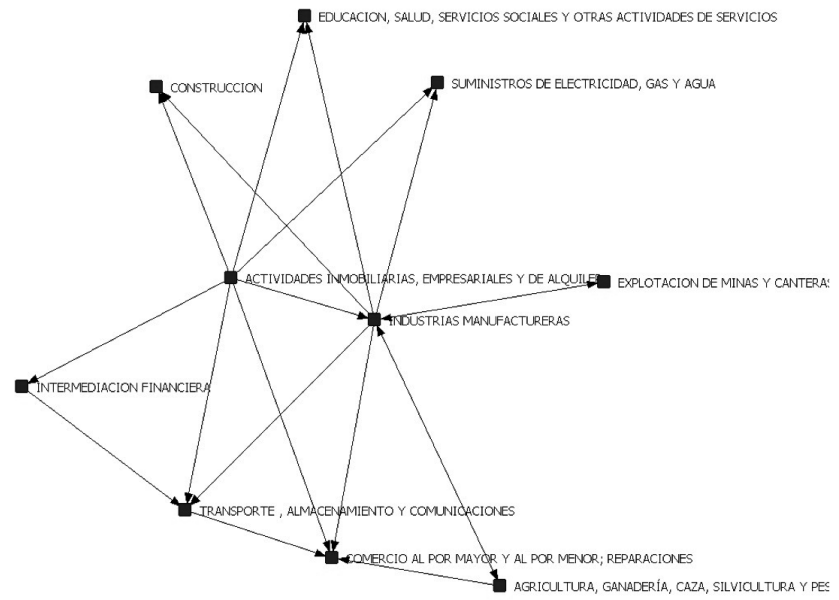

FUENTES: Informe CENICOOP-2013 (Alarcón, 2013) y Borgatti (2002).

\section{Gráfico 4. Grafo de la red de relaciones intersectoriales de la economía cooperativa de Colombia en 2010}

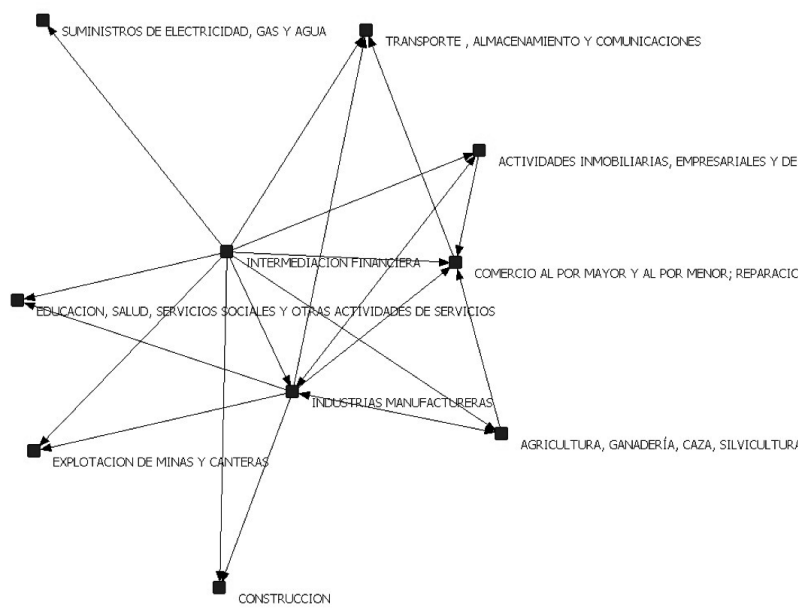

FUENTES: Informe CENICOOP-2013 (Alarcón, 2013) y Borgatti (2002). 
5. La necesidad de generar una línea base sobre el impacto cooperativo como fundamento de la co-construcción de políticas públicas

Los resultados obtenidos por el cooperativismo en la etapa 2003-2010 sirven de punto de partida para discutir cómo optimizar los instrumentos de medición y generar metodologías para el cálculo del impacto cooperativo, dado que la especificidad cooperativa genera que los esfuerzos de identificación, cálculo y análisis deban duplicarse por la doble dimensión de la cooperativa como empresa y como organización que asocia los intereses de personas.

Por otra parte, la identificación de la red de relaciones intersectoriales configuradas por la Economía Nacional y la Economía Cooperativa de Colombia, contribuye a precisar la utilidad social cooperativa y reconocer la estructura de red de relaciones con sus fortalezas y debilidades tal como lo plantea Saz Gil et.al. (2016).

Cabe apuntar que desde 2014 se ha hecho cada vez más difícil homologar los estados financieros a las cuentas que conforman el SCN, dado que los primeros no están disponibles públicamente como antes, se encuentran desagregadas en cinco Superintendencias diferentes y no reportan igual número de variables. Por ello poner en cuestionamiento los métodos de cálculo de impacto es sustantivo para avanzar en la materia, así como también el desarrollo de trabajos que busquen estandarizar variables, pues como afirma Miquel (2015) dichos registros y contabilizaciones "retocados" permiten cuantificar toda la información estadística y ordenarla con calidad estadística.

En adición a lo anterior, no es menos relevante precisar que en la actualidad la economía colombiana está en una particular coyuntura de fomento de cooperativas como consecuencia de las necesidades públicas de propiciar opciones organizacionales en territorios de postconflicto, luego de los Acuerdos de la Habana. En este sentido, son señales claras de un tiempo de particular atención a los temas de políticas públicas en cooperativismo: la creación de la primera cooperativa de la otrora guerrilla de las FARC: Ecomun, la disposición de un Plan para el fomento de la Economía Solidaria, PLANFES y el establecimiento de una mesa de co-construcción de políticas públicas que a mediados de 2017 se está constituyendo. Es aquí donde la concepción de Bretos y Marcuello (2017) puede llegar a tener su evidencia, en tanto el postconflicto ha generado desempleo formal en mercados de trabajo locales y agravio comparativo en términos de las condiciones de los trabajadores, respecto al mercado de trabajo nacional. En ese contexto laboral en el que emergen personas excluidas del mercado laboral formal, junto con otras dinámicas culturales y sociales, se ha llevado a la individualización de las relaciones sociales y la disminución de la cohesión social, de manera que el cooperativismo, con los hábitos de su particular gobernanza, desde una propuesta de institucionalismo económico, tenderían a conseguir la inserción laboral por su diferencial comportamiento respecto a las empresas tradicionales. 
Detrás de estas renovadas acciones por el fomento de la economía solidaria se encuentra el afán por generar políticas públicas que permitan optimizar la incidencia de las cooperativas como organizadoras del territorio y generadoras de convivencia pacífica (Álvarez, 2015). Lo anterior tomando en cuenta que en contextos como el colombiano, las cooperativas tienen presencia en escenarios socioeconómicos y de derechos fundamentales en los cuales la presencia del Estado es prácticamente inexistente y en este contexto las políticas públicas orientadas a combatir la pobreza y la inclusión social ganan en la medida en que incluyen a estas organizaciones en la construcción y reconstrucción del tejido social que conduce a una sociedad más equilibrada e inclusiva (De Sá y Pequito, 2015).

Pero toda política de intervención estatal debe ser articulada con los gremios cooperativos en tanto la evidencia de las CTA demuestra que las organizaciones desarticuladas no logran generar redes de contención a los vaivenes económicos y políticos. Desde ese punto de vista, la experiencia canadiense de las cooperativas de desarrollo rural muestran cómo con el concurso del Estado es posible articular la creación de cooperativas en territorios vulnerables para mantener los servicios de proximidad constituyéndose, tal como lo demuestra Buendía y Coté (2014), en instrumentos de concertación en tanto facilitan la participación de distintos agentes, integran al propio sector cooperativo en estructuras de segundo nivel y orientan su progreso como conocedoras de su territorio, co-construyendo por tanto sus políticas públicas.

\section{Conclusiones}

El cooperativismo alcanza un peso relativo notable en la economía colombiana, el cual se ha duplicado aproximadamente entre 2003 y 2010, llegando a participar del 1,7 por 100 del PIB nacional, y ocupar al 1,4 y 3 por 100 de los asalariados y del empleo colombianos, en un contexto en el que la economía se expandió notablemente y las políticas públicas instrumentalizaron y posteriormente liquidaron a cientos de cooperativas de trabajo asociado. No obstante, la contribución socioeconómica del cooperativismo a la sociedad queda patente.

Sectorialmente, el cooperativismo colombiano se concentra en las ramas de Educación, salud y otros servicios personales y comunitarios, Intermediación Financiera y en Comercio y hostelería que, sumados, llegan a $3 / 4$ de las grandes variables, como el VAB y el empleo. Estos sectores concentran entre el 40 y el 50 por 100 de las mismas variables para la economía nacional. La concentración cooperativa se intensifica en el periodo en las ramas de la Manufactura, la Intermediación Financiera y la Educación, salud y otros servicios personales y comunitarios.

Así, al comparar las relaciones intersectoriales se destacan diferencias estructurales. Si bien las ramas motoras son las mismas en Manufactura, Construcción, Comercio y hostelería y Transportes 


\section{LA DIMENSIÓN SECTORIAL DEL COOPERATIVISMO COLOMBIANO A TRAVÉS DE UN ANÁLISIS DE REDES}

y comunicaciones, resultan ser ramas a las que el sector cooperativo se adapta a lo largo del periodo estudiado, y cuyo peso cooperativo aumenta, especialmente el referido al sector de servicios de Comercio y hostelería.

Paralelamente, la Intermediación Financiera cooperativa, rama de considerable peso en el cooperativismo y en el mismo sector productivo nacional, incrementa sus participaciones tanto en el cooperativismo como en el mismo sector nacional, y mantiene efectos arrastre atrás y adelante por debajo de la media sectorial cooperativa, como ocurre con la rama de Intermediación Financiera nacional.

Como resultado de lo anterior, junto a la diferente estructura sectorial del empleo respecto a la economía nacional, los multiplicadores del empleo cooperativo son mayores a los de la economía colombiana, en congruencia con la menor capitalización respecto a la de la economía nacional -menores tasas de formación bruta de capital sobre el VAB, la tercera parte-. Los arrastres sectoriales del empleo cooperativo tienden a reducirse en menor medida que lo hacen los de la economía nacional, y generan más empleo indirecto por sector que la media nacional, salvo en Suministros de electricidad, gas y agua, Construcción, Comercio y hostelería e Intermediación Financiera, ramas en las que el cooperativismo genera en mayor proporción empleo directo.

Se señala que las redes de relaciones intersectoriales cooperativas son estructuras más centralizadas que las de la economía nacional, sobre todo por el efecto de la Manufactura, la Intermediación Financiera y el sector Agrario, mientras que las ramas de la Manufactura, la de Actividades Inmobiliarias y servicios a empresas, el sector Agrario y la de Transportes y comunicaciones centralizan la red de la economía nacional, la última muy importante en la dinámica de la globalización y que no tiene esa situación en la red cooperativa.

Por otra parte, la densidad de las redes de relaciones intersectoriales cooperativas aumenta hasta el año 2007 y posteriormente cae, iniciándose siempre en niveles inferiores a los nacionales, lo que implica cierta convergencia, que se confirma con cierto incremento de la compactación de la red y de la reducción de la fragmentación. Es decir, que las redes de relaciones intersectoriales de las cooperativas aumentan su cohesión. No obstante, los niveles de compactación y fragmentación respecto a las redes de la economía nacional son menores y mayores, respectivamente.

En consecuencia, los grafos que representan las redes de relaciones intersectoriales de la economía nacional colombiana son más densos y cohesionados que los de su economía cooperativa, pero evolucionan de manera opuesta durante la etapa de estudio, reflejando cierta convergencia.

También se observa el protagonismo intermediador, o poder de intermediación, de la Manufactura en ambas redes, más intenso en la red de la economía nacional, y en menor medida el del Comercio y hostelería, con la misma diferencia de intensidad. A este aspecto relativo a la intermediación cabe añadir la diferencia básica entre las redes cooperativas y las de la economía colombiana, referidas a 
las relaciones intersectoriales: la alta intermediación del sector de Transportes y comunicaciones en la red de la economía nacional, nula en la red cooperativa.

En definitiva, la adaptación del cooperativismo al periodo de expansión económica colombiana, y que este trabajo acotó entre 2003 y 2010, redunda en convergencia en la cohesión de sus redes, aunque se mantiene cierto diferencial en la vertebración a favor de la economía nacional, la cual puede resultar ser un amortiguador en momentos de freno o caída de la actividad económica, al constituir arrastres negativos de menor intensidad en la actividad económica sectorial y en su empleo. Lo cual ha podido contribuir en la etapa 2003-2010 al desarrollo económico y social colombiano, como se ha evidenciado en términos de la contabilidad del crecimiento económico y de otros instrumentos de la economía aplicada.

Estas cifras y el análisis que de ellas se desprende pueden contribuir a fortalecer los estudios iniciales de impacto del cooperativismo en la economía nacional y particularmente pretende fomentarse como medio para el fortalecimiento de la convivencia pacífica en los territorios, expresado a través de la concertación de políticas públicas proclives al estímulo de las formas cooperativas de hacer economía. Más se requiere superar los obstáculos en materia de información y bases de datos y el ejercicio anterior también apunta a generar reflexiones sobre el método que puede desarrollarse al respecto.

\section{Bibliografía}

ALARCÓN, M.A. (2009): "Multiplicadores Input-Output y redes del sistema cooperativo. Posibles impactos de la crisis en la Economía Social lucrativa". En: Actas de XII Jornadas de Investigadores en Economía Social y Cooperativa, 24-26 de junio de 2009, Murcia.

ALARCÓN, M.A. (2011): "Incidencia de la Economía Social y Solidaria en las Cuentas Nacionales. Hacia una identificación de su necesidad y un mapeo de la experiencia europea". En: Álvarez, J.F. (Editor), Innovación y Economía social y solidaria, Barranquilla.

ALARCÓN, M.A., CASTRO, L.M. \& RODRíGUEZ, N. (2013): Metodología para la Construcción del Sistema de Cuentas Nacionales del Sector de Economía Solidaria en Colombia, Documento de trabajo del Proyecto "IMPACTO SOCIAL y ECONÓMICO DEL COOPERATIVISMO EN COLOMBIA", Centro de Investigaciones sobre el Cooperativismo, Bogotá.

ALARCÓN, M.A. (2013): Las Cuentas del Cooperativismo de Colombia. Magnitudes cuantitativas e impacto económico 2003-2010, Documento de Trabajo e Informe Final del Proyecto "IMPACTO SOCIAL y ECONÓMICO DEL COOPERATIVISMO EN COLOMBIA", Centro de Investigaciones sobre el Cooperativismo, Bogotá. 


\section{LA DIMENSIÓN SECTORIAL DEL COOPERATIVISMO COLOMBIANO A TRAVÉS DE UN ANÁLISIS DE REDES}

ALARCÓN, M.A. (2016): "La economía social desde el institucionalismo económico. Evidencia empírica", CIRIEC-España, Revista de Economía Pública, Social y Cooperativa, 86, 61-100.

ÁLVAREZ, J.F. (2015): "Identificación de factores sociales, económicos, políticos y culturales que son dinamizados desde las prácticas de economía solidaria". En: Mora, C. (Ed.), Cooperativismo y convivencia pacífica: análisis de factores y casos, Instituto de Estudios del Ministerio Público, Bogotá.

ÁLVAREZ, J.F. \& GARCÍA-MÜLLER, A. (2013): "Las cooperativas de trabajo asociado y su instrumentalización en Colombia", Revista Idelcoop, 211: 27-43.

BANCO DE LA REPÚBLICA (2018): Buscador de series estadísticas, http://www.banrep.gov.co/es/estadisticas

BAREA, J. \& MONZÓN, J.L. (Dir.) (2006): Manual para la elaboración de las cuentas satélite de las empresas de la Economía social: cooperativas y mutuas, CIRIEC-España, Valencia.

BAREA, J. \& MONZÓN, J.L. (Dir.) (2011): Las cuentas satélite de las cooperativas, mutuas y mutualidades de previsión social en España. Año 2008, INE - CIRIEC-España, Madrid.

BASSI, A. \& VINCENTI, G. (2015): "Toward a new metrics for the evaluation of the social added value of social enterprises", CIRIEC-España, Revista de Economía Pública, Social y Cooperativa, 83, 9-42.

BAVELAS, A. (1948): "A mathematical model for group structure", Human Organizations, 7, 16-30.

BERNAL, A. (2008): Modelo Alternativo de Desarrollo para una Economía Nacional, Plural, Democrática y Participativa, Editorial Oveja Negra-Quintero Editores, Bogotá.

BORGATTI, S.P. (2002): NetDraw: Graph Visualization Software, Analytic Technologies, Harvard.

BORGATTI, S.P., EVERETT, M.G. \& FREEMAN, L.C. (2002): Ucinet for Windows: Software for Social Network Analysis, Analytic Technologies, Harvard, Massachusetts.

BRETOS, I. \& MARCUELLO, C. (2017): "Revisiting Globalization Challenges And Opportunities In The Development Of Cooperatives", Annals of Public and Cooperative Economics, 88:1, 47-73.

CLARK, C. (1949): "Theory of Economic Growth", Econometrica, Vol. 17, Supplement: Report of the Washington Meeting, pp. 112-116.

CLARK, C. (1980): Las Condiciones del Progreso Económico, Alianza Universidad, Madrid. Traducción del texto de Macmillan, Londres de 1940, The Conditions of Economic Progress.

BUENDÍA-MARTÍNEZ, I. \& COTÉ, A. (2014): "Desarrollo territorial rural y cooperativas: un análisis desde las políticas públicas", Cuadernos de Desarrollo Rural, 11 (74): 35-54.

CUÑAT, R. (2015): "Las redes como factor clave para la consolidación de nuevas cooperativas de trabajo asociado", REVESCO, Revista de Estudios Cooperativos, 119, Tercer Cuatrimestre, $26-52$.

DANE (1986): Metodología de las Cuentas Nacionales de Colombia, Bogotá. 
DANE (2005): Caracterización de las instituciones sin fines de lucro en Colombia, Dirección de Síntesis y Cuentas Nacionales, Bogotá.

DANE (2008): Metodología de las Cuentas Nacionales de Colombia - Cuentas de los Sectores Institucionales, Documento de Trabajo para el Proyecto Andestad, Bogotá.

DE SÁ, J. \& PEQUITO, C. (2015): Capital social, economia social e qualidade da democracia em Portugal, Editorial Campo Da Comunicação, Lisboa.

FREEMAN, L.C. (1979): "Centrality in Social Networks. Conceptual Clarification", Social Networks, 1:215-239.

HANNEMAN, R.A. \& RIDDLE, M. (2005): Introduction to social network methods, University of California, Riverside. Http://faculty.ucr.edu/ hanneman/

MIQUEL, B. (2015): "La medición del bienestar económico a través de las macromagnitudes de la contabilidad nacional", CIRIEC-España, Revista de Economía Pública, Social y Cooperativa, 85, 245286.

NAVARRA, C. (2016): "Employment Stabilization Inside Firms: An Empirical Investigation Of Worker Cooperatives", Annals of Public and Cooperative Economics, 87:2, 563-585.

NDIEGE , B.O., QUIN, X., MASSAMBU, D. \& NTOWO, E. (2016): "Analysis Of The Possibilities For Expansion Of Services In Tanzanian Savings And Credits Co-Operative Societies: Learning From Economies Of Scale", Annals of Public and Cooperative Economics, 87:2, 239-255.

RASMUSSEN, P.N. (1956): Studies in Intersectorial Relations, North-Holland, Amsterdan.

SCOTT, J. (1991): Social network analysis: a handbook, Sage, Londres.

RODRÍGUEZ, N. (1993): Sistema de Información que Permita Obtener la Matriz de Insumo- Producto en las Organizaciones Cooperativas, Universidad Central.

RODRÍGUEZ, N., SÁNCHEZ, C., ÁLVAREZ, J.F. \& FAJARDO, M.A. (2016): Medición del impacto socioeconómico del cooperativismo: propuesta para su cálculo, Instituto de Estudios del Ministerio Público, Bogotá.

SALA, M., FARRÉ, M. \& TORRES, T. (2014): "Un análisis del comportamiento cíclico de las cooperativas y sociedades laborales españolas y su relación con la actividad económica", REVESCO, Revista de Estudios Cooperativos, 115 - Segundo Cuatrimestre, 7-29.

SAZ-GIL, I., ALMAGUER-KALIXTO, P. \& GÓMEZ-QUINTERO, J.D. (2016): "Capital social y redes sociales: análisis del Tercer Sector en contextos rurales", CIRIEC-España, Revista de Economía Pública, Social y Cooperativa, 86, 123-154.

UNITED NATIONS (2003): Handbook on Non-Profit Institutions in the System of National Accounts, United Nations, New York. 